\title{
SINGULARITIES OF IMPLICIT DIFFERENTIAL SYSTEMS AND THEIR INTEGRABILITY
}

\author{
TAKUO FUKUDA \\ Department of Mathematics, College of Humanities and Sciences \\ Sakurajousui 3-25-40, Setagaya-ku, Tokyo, Japan \\ E-mail: fukuda@math.chs.nihon-u.ac.jp
}

STANISEAW JANECZKO

Institute of Mathematics, Polish Academy of Sciences

Sniadeckich 8, P.O.Box 21, 00-956 Warszawa, Poland;

Faculty of Mathematics and Information Science, Warsaw University of Technology

Pl. Politechniki 1, 00-661 Warszawa, Poland

E-mail: janeczko@ise.pw.edu.pl

1. Introduction. If $P$ is a smooth manifold and $V: P \rightarrow T P$ is a vector field on $P$, i.e. a section of the tangent bundle $\pi_{1}: T P \rightarrow P$, then the local integrability of $V$ is a characteristic property, especially for smooth sections $V$. Traditionally the local integrability of a vector field $V$ (not necessary continuous) on $P$ is defined as an existence at each point $p$ of the domain of $V$ a $C^{1}$-curve $\gamma:(-\varepsilon, \varepsilon) \rightarrow P$ (for some $\varepsilon>0$ ), such that $\gamma(0)=p$ and $\frac{\partial \gamma}{\partial t}(t)=V(\gamma(t))$ for all $t \in(-\varepsilon, \varepsilon)$. The curve $\gamma$ is called an integral curve of $V$ with initial value $p$. We may assume that the existence of such $\gamma$ for $V$ is not an exceptional property, and that for each $p \in P$ there exist a neighbourhood $U$ of $p$ and $\varepsilon>0$ such that the mapping $U \times(-\varepsilon, \varepsilon) \ni(\bar{p}, t) \mapsto \gamma_{\bar{p}}(t), \gamma_{\bar{p}}(0)=\bar{p}$, is defined and at least continuous. For smooth, compactly supported $V$ this mapping forms a one parameter group of transformations of $P$.

When we replace $V$ by a smooth embedded manifold $M$ into $T P$, then the mentioned integrability property becomes rather the restrictive exceptional property for $M$, especially in the critical point set of the projection $\left.\pi_{1}\right|_{M}: M \rightarrow P$. In general, such systems are called implicit differential systems (equations) of order 1 , and were studied by several authors: in connection to the non-continuous integrability property by [21], [7]. The local

2000 Mathematics Subject Classification: Primary 57R45; Secondary 58C35, 70H05, 34A26.

Research partially supported by KBN grant No. 2 P03A 02017 and Special Program "Dynamics of Complex Systems" Warsaw Univ. of Technology.

The paper is in final form and no version of it will be published elsewhere. 
equivalences of singularities of such systems were studied (among others) by [22], [4], [5], [20]. The Hamiltonian implicit case which is especially addressed to mathematical physics was introduced to mathematics by P. A. M. Dirac [6], investigated also by [16], [2], [10].

The local smooth integrability property is important for understanding the local dynamics of the system and the structure of the induced transformation groups. However the integrability property is not a usual property in the implicit case. Then a natural question which arises, is to characterize those implicit systems $M$ which are integrable, i.e. for each point of $M$ there is a local integral curve passing through. Another natural question is to understand the reason for non-integrability - the local topological structure of the strata of non-integrable points.

The purpose of this paper is threefold: First we provide necessary and sufficient conditions of integrability for various classes of implicit systems. Second we formulate and investigate the problem of integrability for implicit Hamiltonian systems as a specialized class of implicit systems, and third we search for the generic peculiarities of non-integrable points.

In Sections 2, 3 and 4 we formulate the integrability problem, consider the twodimensional fold and cusp cases and prove the first part of the main results of the paper: the smooth integrability necessary and sufficient conditions for the generic (in the sense of J. Mather) differential systems (Theorems 3.1 and 3.2). In Section 5 the crucial examples and counterexamples of various integrability and non-integrability situations are constructed. Passing to real analytic category, in Section 6, we extend J. Mather's results weakening his transversality condition to the irreducibility of determinants. This result is formulated in Theorems 6.1 and 6.2. Along the road to this result we prove several applicable properties of prime ideals having the property of zeroes. Specialization of our differential systems to the Hamiltonian ones, which was a part of the motivation for this paper, is done in Section 7. In this case we apply the main results of the previous sections and obtain the necessary and sufficient conditions for integrability of implicit Hamiltonian systems. Here we develop the computable formula for the integrability check in case of P. A. M. Dirac generalized Hamiltonian systems. Finally Section 8 is devoted to the non-integrable points of differential systems. We prove here (Theorems 8.1, 8.2) that the non-integrable points are isolated generically in the sense of J. C. Tougeron.

2. The integrability problem. Let $M$ be a smooth submanifold of $T \mathbf{R}^{n}, M \subset$ $T \mathbf{R}^{n} \equiv \mathbf{R}^{n} \times \mathbf{R}^{n}, \operatorname{dim} M=m . M$ is considered as a first order differential system.

Definition 2.1. A point $(p, q) \in M \subset T \mathbf{R}^{n} \equiv \mathbf{R}^{n} \times \mathbf{R}^{n}$ is called an integrable point of $M$ if there exists a smooth curve $\gamma:(-\varepsilon, \varepsilon) \rightarrow \mathbf{R}^{n}$ such that

$$
\gamma(0)=p, \quad \gamma^{\prime}(0)=q \quad \text { and } \quad\left(\gamma(t), \gamma^{\prime}(t)\right) \in M \text { for all } t \in(-\varepsilon, \varepsilon) .
$$

The manifold $M$ is called an integrable manifold if $M$ consists only of integrable points.

Now we have a natural question.

Problem 2.1. Find a necessary and sufficient condition for a manifold $M \subset T \mathbf{R}^{n}$ to be integrable. 
We immediately find a necessary condition as follows. Let $\pi_{1}: T \mathbf{R}^{n} \equiv \mathbf{R}^{n} \times \mathbf{R}^{n} \rightarrow \mathbf{R}^{n}$ be the projection to the first factor:

$$
\pi_{1}: \mathbf{R}^{n} \times \mathbf{R}^{n} \rightarrow \mathbf{R}^{n}, \quad \pi_{1}(p, q)=p, \quad \text { tangent bundle projection. }
$$

Let $(p, q) \in M$ be an integrable point of $M$. Then from the definition there exists a smooth curve $\gamma:(-\varepsilon, \varepsilon) \rightarrow \mathbf{R}^{n}$ such that

$$
\gamma(0)=p, \quad \gamma^{\prime}(0)=q \quad \text { and } \quad\left(\gamma(t), \gamma^{\prime}(t)\right) \in M
$$

Then we have

$$
q=\gamma^{\prime}(0)=\frac{d\left(\pi_{1}\left(\gamma, \gamma^{\prime}\right)\right)}{d t}(0)=d\left(\left.\pi_{1}\right|_{M}\right)_{(p, q)}\left(\gamma^{\prime}(0), \gamma^{\prime \prime}(0)\right) \in d\left(\left.\pi_{1}\right|_{M}\right)_{(p, q)}\left(T_{(p, q)} M\right) .
$$

Thus an immediate necessary condition for a point $(p, q) \in M$ to be integrable is that

$$
q \in d\left(\left.\pi_{1}\right|_{M}\right)_{(p, q)}\left(T_{(p, q)} M\right) .
$$

In what follows we will call this condition tangential integrability condition.

We can ask whether this condition is also a sufficient condition for a submanifold $M$ to be integrable. Although the answer for this question is negative, there is a wide class of submanifolds of $T \mathbf{R}^{n}$ for which the tangential integrability condition is also sufficient. An example of the submanifold $M$ for which the tangential integrability condition is fulfilled but $M$ is not integrable is given in Section 5 (see Example 5.1).

To come closer to our problem we explain some sufficient conditions for a submanifold $M$ of $T \mathbf{R}^{n}$ to be integrable. One of them plays an important role in the proofs of our main theorems. At first we have the following two immediate lemmas.

LEMMA 2.1. If a submanifold $M \subset T \mathbf{R}^{n}$ is a finite union of integrable submanifolds $M_{i} \subset T \mathbf{R}^{n}$ :

$$
M=\bigcup_{i} M_{i}, \quad M_{i} \text { integrable, }
$$

then $M$ is integrable.

LEMma 2.2. Let $M \subset T \mathbf{R}^{n}$ be a submanifold satisfying tangential integrability condition.

1) If $\left.\pi_{1}\right|_{M}: M \rightarrow \mathbf{R}^{n}$ is either an embedding or a submersion, then $M$ is integrable.

2) If $\pi_{1}(M)$ is a submanifold of $\mathbf{R}^{n}$ and $\left.\pi_{1}\right|_{M}: M \rightarrow \pi_{1}(M)$ is a submersion, then $M$ is integrable.

Since our problem is a local one, we may suppose that the manifold $M$ under consideration is the image of an embedding of $\mathbf{R}^{m}$ :

$$
M=(f, g)\left(\mathbf{R}^{m}\right), \quad(f, g): \mathbf{R}^{m} \rightarrow T \mathbf{R}^{n}=\mathbf{R}^{n} \times \mathbf{R}^{n} \text { is an embedding. }
$$

Let $J f(x)$ denote the Jacobian matrix of $f=\left(f_{1}, \ldots, f_{n}\right)$ at $x=\left(x_{1}, \ldots, x_{m}\right) \in \mathbf{R}^{m}$,

$$
J f(x)=\left(\begin{array}{ccc}
\frac{\partial f_{1}}{\partial x_{1}}(x) & \ldots & \frac{\partial f_{1}}{\partial x_{m}}(x) \\
\vdots & \vdots & \vdots \\
\frac{\partial f_{n}}{\partial x_{1}}(x) & \ldots & \frac{\partial f_{n}}{\partial x_{m}}(x)
\end{array}\right) .
$$


Lemma 2.3. Let $M=(f, g)\left(\mathbf{R}^{m}\right) \subset T \mathbf{R}^{n}$ be as above. Then $M$ satisfies the tangential integrability condition if and only if

$$
g(x) \in J f(x)\left(\mathbf{R}^{m}\right) \quad \text { for every } x \in \mathbf{R}^{m} .
$$

Proof. Let $(p, q)=(f(x), g(x))$ and let $\pi_{1}: T \mathbf{R}^{n} \equiv \mathbf{R}^{n} \times \mathbf{R}^{n} \rightarrow \mathbf{R}^{n}$ be the projection of the tangent bundle. Since $d\left(\left.\pi_{1}\right|_{M}\right)\left(T_{(p, q)} M\right)=J f(x)\left(\mathbf{R}^{m}\right)$, we see that

$$
g(x) \in d\left(\left.\pi_{1}\right|_{M}\right)\left(T_{(p, q)} M\right)
$$

if and only if $g(x) \in J f(x)\left(\mathbf{R}^{m}\right)$.

LEMMA 2.4. Let $M=(f, g)\left(\mathbf{R}^{m}\right)$, where

$$
(f, g): \mathbf{R}^{m} \rightarrow T \mathbf{R}^{n}=\mathbf{R}^{n} \times \mathbf{R}^{n} \text { is an embedding. }
$$

If there exist smooth local functions $a_{1}\left(x_{1}, \ldots, x_{m}\right), \ldots, a_{m}\left(x_{1}, \ldots, x_{m}\right)$ such that

$$
\left(\begin{array}{c}
g_{1}(x) \\
g_{2}(x) \\
\vdots \\
g_{n}(x)
\end{array}\right)=\left(\begin{array}{ccc}
\frac{\partial f_{1}}{\partial x_{1}}(x) & \ldots & \frac{\partial f_{1}}{\partial x_{m}}(x) \\
\frac{\partial f_{2}}{\partial x_{1}}(x) & \ldots & \frac{\partial f_{2}}{\partial x_{m}}(x) \\
\vdots & \vdots & \vdots \\
\frac{\partial f_{n}}{\partial x_{1}}(x) & \ldots & \frac{\partial f_{n}}{\partial x_{m}}(x)
\end{array}\right)\left(\begin{array}{c}
a_{1}(x) \\
a_{2}(x) \\
\vdots \\
a_{m}(x)
\end{array}\right)
$$

then $M$ is integrable.

Proof. Take any point $\left(p_{0}, q_{0}\right)=(f, g)\left(x_{0}\right) \in M$. Consider the vector field

$$
\xi(x)=a_{1}(x)\left(\frac{\partial}{\partial x_{1}}\right)_{x}+a_{2}(x)\left(\frac{\partial}{\partial x_{2}}\right)_{x}+\ldots+a_{m}(x)\left(\frac{\partial}{\partial x_{m}}\right)_{x}
$$

defined on $\mathbf{R}^{m}$. Consider an integral curve

$$
\widetilde{\gamma}:(-\varepsilon, \varepsilon) \rightarrow \mathbf{R}^{m} \text { of } \xi \text { such that } \widetilde{\gamma}(0)=x_{0}
$$

and consider the curve $\gamma:(-\varepsilon, \varepsilon) \rightarrow \mathbf{R}^{n}, \gamma(t)=f(\widetilde{\gamma}(t))$.

Then the point $\left(p_{0}, q_{0}\right)=(f, g)\left(x_{0}\right) \in M$ is an integrable point of $M$ with the curve $\gamma(t)$. This can be seen as follows. Since

$$
\frac{d \widetilde{\gamma}}{d t}(t)=a(\widetilde{\gamma}(t))=\left(a_{1}(\widetilde{\gamma}(t)), \ldots, a_{m}(\widetilde{\gamma}(t))\right)
$$

we have

$$
\begin{aligned}
\frac{d \gamma}{d t}(t)=\frac{d(f \circ \widetilde{\gamma})(t)}{d t} & =J f(\widetilde{\gamma}(t)) \cdot \frac{d \widetilde{\gamma}}{d t}(t) \\
= & \left(\begin{array}{ccc}
\frac{\partial f_{1}}{\partial x_{1}}(\widetilde{\gamma}(t)) & \ldots & \frac{\partial f_{1}}{\partial x_{m}}(\widetilde{\gamma}(t)) \\
\frac{\partial f_{2}}{\partial x_{1}}(\widetilde{\gamma}(t)) & \ldots & \frac{\partial f_{2}}{\partial x_{m}}(\widetilde{\gamma}(t)) \\
\vdots & \vdots & \vdots \\
\frac{\partial f_{n}}{\partial x_{1}}(\widetilde{\gamma}(t)) & \ldots & \frac{\partial f_{n}}{\partial x_{m}}(\widetilde{\gamma}(t))
\end{array}\right)\left(\begin{array}{c}
a_{1}(\widetilde{\gamma}(t)) \\
a_{2}(\widetilde{\gamma}(t)) \\
\vdots \\
a_{m}(\widetilde{\gamma}(t))
\end{array}\right)=\left(\begin{array}{c}
g_{1}(\widetilde{\gamma}(t)) \\
g_{2}(\widetilde{\gamma}(t)) \\
\vdots \\
g_{n}(\widetilde{\gamma}(t))
\end{array}\right) .
\end{aligned}
$$

Hence we have

$$
\left(\gamma(t), \gamma^{\prime}(t)\right)=(f(\widetilde{\gamma}(t)), g(\widetilde{\gamma}(t))) \in M=(f, g)\left(\mathbf{R}^{m}\right) \quad \text { and } \quad\left(\gamma(0), \gamma^{\prime}(0)\right)=\left(p_{0}, q_{0}\right) .
$$

Thus $\left(p_{0}, q_{0}\right)$ is an integrable point of $M$. 
Corollary 2.1. Let $f: \mathbf{R}^{m} \rightarrow \mathbf{R}^{n}$ be a smooth mapping with

$$
\operatorname{rank} d f_{x} \geq \frac{m}{2} \quad \text { for all } x \in \mathbf{R}^{m} \text {. }
$$

Then there exists (always) a smooth mapping $g: \mathbf{R}^{m} \rightarrow \mathbf{R}^{n}$ such that $(f, g): \mathbf{R}^{m} \rightarrow$ $\mathbf{R}^{n} \times \mathbf{R}^{n}$ is an embedding and $M=(f, g)\left(\mathbf{R}^{m}\right)$ is an integrable manifold.

Proof. Since we consider the local problem and since

$$
r=\operatorname{rank} d f_{x}: T_{x}\left(\mathbf{R}^{m}\right) \rightarrow T_{f(x)} \mathbf{R}^{n} \geq \frac{m}{2} \text { for all } x \in \mathbf{R}^{m},
$$

we may choose local coordinates such that $f$ is of the form

$$
f\left(x_{1}, \ldots, x_{m}\right)=\left(x_{1}, \ldots, x_{r}, f_{r+1}\left(x_{1}, \ldots, x_{m}\right), \ldots, f_{n}\left(x_{1}, \ldots, x_{m}\right)\right) .
$$

Let $a_{1}(x), \ldots, a_{n}(x)$ be the smooth functions chosen in the form

$$
a_{1}(x)=x_{r+1}, \ldots, a_{m-r}(x)=x_{m} \quad \text { and } \quad a_{m-r+1}(x), \ldots, a_{n}(x) \text { being arbitrary. }
$$

Consider a mapping $g=\left(g_{1}, \ldots, g_{n}\right): \mathbf{R}^{m} \rightarrow \mathbf{R}^{n}$ defined by equation (1). Then $(f, g)$ : $\mathbf{R}^{m} \rightarrow \mathbf{R}^{n} \times \mathbf{R}^{n}$ is an embedding and from Lemma 2.4 we see that $M=(f, g)\left(\mathbf{R}^{m}\right)$ is an integrable manifold.

REMARK 2.1. Now we came to the natural question: Does some pathology of the critical point set on $M$ imply the non-integrability? To some extent the answer is positive. We may formulate the following supposition: if $f: \mathbf{R}^{m} \rightarrow \mathbf{R}^{n}$ is stable, then for any $g: \mathbf{R}^{m} \rightarrow \mathbf{R}^{n}$ such that $(f, g): \mathbf{R}^{m} \rightarrow \mathbf{R}^{n} \times \mathbf{R}^{n}$ is an embedding and $M=(f, g)\left(\mathbf{R}^{m}\right)$ satisfies tangential integrability condition, $M$ has to be integrable. Therefore if this conjecture is true, it implies that if $M$ is not integrable, then $f$ is not stable, and therefore $\left.\pi_{1}\right|_{M}: M \rightarrow \mathbf{R}^{n}$ is not a stable map. Let us remind here that the two maps $f: \mathbf{R}^{m} \rightarrow \mathbf{R}^{n}$ and $\left.\pi_{1}\right|_{M}: M \rightarrow \mathbf{R}^{n}$ are right equivalent in J. Mather's sense, since $(f, g): \mathbf{R}^{m} \rightarrow M=(f, g)\left(\mathbf{R}^{m}\right)$ is a diffeomorphism. Thus the singular point set is not the one of a stable map. On the other hand, Corollary 2.1 says that if

$$
\operatorname{rank}\left(\left.d \pi_{1}\right|_{M}\right): T_{(p, q)}(M) \rightarrow T_{p}\left(\mathbf{R}^{n}\right) \geq \frac{m}{2},
$$

then no matter how much pathological its singularity is, with a good partner $g$, $M=(f, g)\left(\mathbf{R}^{m}\right)$ becomes integrable.

To complete our sufficient condition formulated in Lemma 2.4 we present Example 5.2 in Section 5, showing that the condition of existence of smooth solution of the linear equation in Lemma 2.4 is not a necessary condition for integrability.

3. Generic manifolds are integrable. Now we prove that the tangential integrability condition is also sufficient for a class of manifolds, which we call the generic manifolds and which is much wider than the class of manifolds $M \subset T \mathbf{R}^{n}=\mathbf{R}^{n} \times \mathbf{R}^{n}$ such that the projections $\left.\pi_{1}\right|_{M}: M \rightarrow \mathbf{R}^{n}$ are $C^{\infty}$-stable.

We saw in Lemma 2.4 that if the linear equation (1) has a smooth solution, then the manifold $M$ is integrable. For a condition that a linear equation (1) has a smooth solution, we have the theorem of J. Mather ([13]). Combining Lemma 2.4 and J. Mather's theorem we obtain a sufficient condition for a manifold to be integrable. 
Let $J^{1}\left(M, \mathbf{R}^{n}\right)$ be the 1-jet bundle of 1-jets of maps of $M$ into $\mathbf{R}^{n}$, and let $\Sigma_{r}\left(M, \mathbf{R}^{n}\right)$ be the subset of all 1-jets $j^{1} f(x) \in J^{1}\left(M, \mathbf{R}^{n}\right)$ such that the rank of the differential $d f_{x}: T_{x}(M) \rightarrow T_{f(x)} \mathbf{R}^{n}$ at $x \in M$ is $r$. Then $\Sigma_{r}\left(M, \mathbf{R}^{n}\right)$ is a submanifold of $J^{1}\left(M, \mathbf{R}^{n}\right)$ of codimension $(n-r) \times(m-r)$, where $m=\operatorname{dim} M$.

Definition 3.1 (following J. Mather [13]). A manifold $M \subset T \mathbf{R}^{n}=\mathbf{R}^{n} \times \mathbf{R}^{n}$ is called a generic submanifold of $T \mathbf{R}^{n}$ if the 1 -jet extension $j^{1}\left(\left.\pi_{1}\right|_{M}\right): M \rightarrow J^{1}\left(M, \mathbf{R}^{n}\right)$ is transversal to all $\Sigma_{r}\left(M, \mathbf{R}^{n}\right), r=0,1, \ldots, \min (m, n)$.

Now we can formulate our first main theorem.

TheOREM 3.1. A generic submanifold $M$ of $T \mathbf{R}^{n}$ is integrable if and only if it satisfies tangential integrability condition. Thus tangential integrability condition is sufficient for generic submanifolds of $T \mathbf{R}^{n}$.

REMARK 3.1. Remark that generic submanifolds form a very wide class in the set of all submanifolds of $T \mathbf{R}^{n}$. This can be seen as follows. Since our problem is a local one, we may suppose that the manifold $M$ under consideration is the image of an embedding of $\mathbf{R}^{m}$ into $T \mathbf{R}^{n}$ :

$$
M=(f, g)\left(\mathbf{R}^{m}\right), \quad(f, g): \mathbf{R}^{m} \rightarrow T \mathbf{R}^{n}=\mathbf{R}^{n} \times \mathbf{R}^{n} \text { an embedding. }
$$

Then, the 1-jet extension $j^{1}\left(\left.\pi_{1}\right|_{M}\right): M \rightarrow J^{1}\left(M, \mathbf{R}^{n}\right)$ is transversal to $\Sigma_{r}\left(M, \mathbf{R}^{n}\right)$ if and only if the 1-jet extension $j^{1} f: \mathbf{R}^{m} \rightarrow J^{1}\left(\mathbf{R}^{m}, \mathbf{R}^{n}\right)$ is transversal to $\Sigma_{r}\left(\mathbf{R}^{m}, \mathbf{R}^{n}\right)$. By Thom's transversality theorem, the set of smooth maps $f: \mathbf{R}^{m} \rightarrow \mathbf{R}^{n}$ such that $j^{1} f: \mathbf{R}^{m} \rightarrow J^{1}\left(\mathbf{R}^{m}, \mathbf{R}^{n}\right)$ is transversal to all $\Sigma_{r}\left(\mathbf{R}^{m}, \mathbf{R}^{n}\right), r=0,1, \ldots, \min (m, n)$ is an open dense set in the set of smooth maps of $\mathbf{R}^{m}$ into $\mathbf{R}^{n}$. Thus generic submanifolds form a very wide class in the set of all submanifolds of $T \mathbf{R}^{n}$.

Now we reformulate our problem in more accessible terms.

Definition 3.2. A smooth map $g: \mathbf{R}^{m} \rightarrow \mathbf{R}^{n}$ is said to be tangent to a smooth map $f: \mathbf{R}^{m} \rightarrow \mathbf{R}^{n}$ if the pair $(f, g): \mathbf{R}^{m} \rightarrow T \mathbf{R}^{n}=\mathbf{R}^{n} \times \mathbf{R}^{n}$ forms an embedding and the linear equation (1) has a solution $\left(a_{1}(x), a_{2}(x), \ldots, a_{n}(x)\right)$ for every point $x \in \mathbf{R}^{m}$.

We see that the tangential integrability condition becomes exactly the tangency of the smooth map $g: \mathbf{R}^{m} \rightarrow \mathbf{R}^{n}$ to the smooth map $f: \mathbf{R}^{m} \rightarrow \mathbf{R}^{n}$, defining the embedding $(f, g): \mathbf{R}^{m} \rightarrow T \mathbf{R}^{n}=\mathbf{R}^{n} \times \mathbf{R}^{n}$ of $M$. If we treat $g$ as a vector field $g(x)=\sum_{j=1}^{n} g_{j}(x) \frac{\partial}{\partial p_{j}}$ then the tangency condition means that the equation $\left(f_{*} X\right)(x)=g(x)$ has a solution $X(x)=\sum_{i=1}^{m} X_{i}(x) \frac{\partial}{\partial x_{i}}$ for any $x \in \mathbf{R}^{m}$.

Now we come to the following problem.

Problem 3.1. Find conditions to be posed on a smooth map $f: \mathbf{R}^{m} \rightarrow \mathbf{R}^{n}$ so that the image $M=(f, g)\left(\mathbf{R}^{m}\right)$ be an integrable manifold for every smooth map $g: \mathbf{R}^{m} \rightarrow \mathbf{R}^{n}$ tangent to $f$.

We can also reformulate this problem in other words as follows:

Find conditions to be posed on a smooth map $f: \mathbf{R}^{m} \rightarrow \mathbf{R}^{n}$ so that the linear equation (1) has a smooth solution $\left(a_{1}(x), a_{2}(x), \ldots, a_{m}(x)\right)$ for every smooth map $g: \mathbf{R}^{m} \rightarrow \mathbf{R}^{n}$ tangent to $f$. 
The result of J. Mather [13] concerning solutions of linear equation helps to give a good answer to this problem. In the rest of this section, we will follow the notation from [13]. Consider an $n \times m$ matrix $A(x)$ of smooth function-germs : $\left(\mathbf{R}^{k}, 0\right) \rightarrow \mathbf{R}$

and a column vector

$$
A(x)=\left(\begin{array}{cccc}
a_{11}(x) & a_{12}(x) & \ldots & a_{1 m}(x) \\
a_{21}(x) & a_{22}(x) & \ldots & a_{2 m}(x) \\
\vdots & \vdots & \vdots & \vdots \\
a_{n 1}(x) & a_{n 2}(x) & \ldots & a_{n m}(x)
\end{array}\right)
$$

$$
g(x)=\left(\begin{array}{c}
g_{1}(x) \\
\vdots \\
g_{n}(x)
\end{array}\right)
$$

where $a_{i j}(x)$ and $g_{i}$ are smooth function-germs $\mathbf{R}^{m} \rightarrow \mathbf{R}$. Consider the linear equation

$$
A(x) b=g(x)
$$

where $b$ is a column vector of length $m$.

Let $E(n, m)$ denote the space of $n \times m$ matrices of real numbers. Let $S_{r}$ denote the subset of $E(n, m)$ consisting of matrices of rank $r$. Then $S_{r}$ is a submanifold of codimension $(n-r) \times(m-r)$ in $E(n, m)$.

Definition 3.3. An $n \times m$ matrix $A(x)$ of smooth function-germs is said to be generic if $A:\left(\mathbf{R}^{k}, 0\right) \rightarrow E(n, m)$ is transversal to all $\Sigma_{r}, r=0,1, \ldots, \min (n, m)$.

J. Mather's Theorem $([13])$. Let $A:\left(\mathbf{R}^{k}, 0\right) \rightarrow E(n, m)$ be a smooth map-germ and let $g:\left(\mathbf{R}^{k}, 0\right) \rightarrow \mathbf{R}^{n}$ be a smooth map-germ such that the linear equation (2) has a solution $b(x)$ for every $x \in \mathbf{R}^{k}$ close to the origin $0 \in \mathbf{R}^{k}$. If the map-germ $A:\left(\mathbf{R}^{k}, 0\right) \rightarrow$ $E(n, m)$ is generic, i.e. is transversal to all $\Sigma_{r}, r=0,1, \ldots, \min (n, m)$, at every point $x \in \mathbf{R}^{k}$ close to the origin $0 \in \mathbf{R}^{k}$, then the equation (2) has a local smooth solution $b(x)$ defined in a neighbourhood of the origin $0 \in \mathbf{R}^{k}$.

4. Proof of main theorems. Since our problem is local, we use the notation of germs. Let $f: \mathbf{R}^{m} \rightarrow \mathbf{R}^{n}$ be a smooth mapping. We may suppose that $f(0)=0$ without loss of generality. Denote by $f:\left(\mathbf{R}^{m}, 0\right) \rightarrow\left(\mathbf{R}^{n}, 0\right)$ its map-germ at $0 \in \mathbf{R}^{m}$. Consider the map

$$
J f: \mathbf{R}^{m} \rightarrow E(n, m) \text { given by } J f(x)=\text { the Jacobian matrix at } x \in \mathbf{R}^{m}
$$

and its germ

$$
J f:\left(\mathbf{R}^{m}, 0\right) \rightarrow E(n, m) \text { at } 0 \in \mathbf{R}^{m} .
$$

Now we can reformulate Theorem 3.1 in the following form.

TheOrem 4.1. Let the rank of $J f(0)$ be equal to $r$. If $J f:\left(\mathbf{R}^{m}, 0\right) \rightarrow E(n, m)$ is transversal to $S_{r}$ at $0 \in \mathbf{R}^{m}$, then, for any smooth map-germ $g:\left(\mathbf{R}^{m}, 0\right) \rightarrow \mathbf{R}^{n}$ tangent to $f:\left(\mathbf{R}^{m}, 0\right) \rightarrow\left(\mathbf{R}^{n}, 0\right)$, the germ of the manifold

$$
M=(f, g)\left(\mathbf{R}^{m}\right) \subset T \mathbf{R}^{n}=\mathbf{R}^{n} \times \mathbf{R}^{n}
$$

at $(0, g(0))$ is integrable. 
Now we have immediately:

Corollary 4.1. Let $f:\left(\mathbf{R}^{m}, 0\right) \rightarrow\left(\mathbf{R}^{n}, 0\right)$ be a $C^{\infty}$-stable map-germ. Then for any smooth map-germ $g:\left(\mathbf{R}^{m}, 0\right) \rightarrow \mathbf{R}^{n}$ tangent to $f:\left(\mathbf{R}^{m}, 0\right) \rightarrow\left(\mathbf{R}^{n}, 0\right)$, the manifold-germ $\left(M=(f, g)\left(\mathbf{R}^{m}\right),(f(0)=0, g(0))\right)$ is integrable.

Proof. Stable maps are transversal to every $\mathcal{A}$-orbit in the jet spaces (cf. [12], [14]), so they are also transversal to an $S_{r}, r=0,1, \ldots, \min (m, n)$. Thus from the Theorem 3.1, the germ of the manifold $M=(f, g)\left(\mathbf{R}^{m}\right)$ at $(0, g(0))$ is integrable.

The main theorems 3.1 and 4.1 follow from J. Mather's Theorem and the previous Lemma 2.4.

Proof of Theorem 4.1. Let the rank of $J f(0)$ be equal to $r$. Suppose that $J f$ : $\left(\mathbf{R}^{m}, 0\right) \rightarrow E(n, m)$ is transversal to $S_{r}$ at $0 \in \mathbf{R}^{m}$. Since the stratification

$$
\left\{S_{i}\right\}_{i=0}^{\min (m, n)}
$$

of $E(n, m)$ satisfies Whitney's condition (a), this implies that $J f:\left(\mathbf{R}^{m}, 0\right) \rightarrow E(n, m)$ is transversal to all $\Sigma_{i}\left(\mathbf{R}^{m}, \mathbf{R}^{n}\right)$ at every point near the origin $0 \in \mathbf{R}^{m}$. Thus from J. Mather's Theorem, for any smooth map-germ $g:\left(\mathbf{R}^{m}, 0\right) \rightarrow \mathbf{R}^{n}$ tangent to $f$ : $\left(\mathbf{R}^{m}, 0\right) \rightarrow\left(\mathbf{R}^{n}, 0\right)$, the linear equation $J f(x) b=g(x)$ has a smooth solution $b(x)$ defined in a neighbourhood of the origin $0 \in \mathbf{R}^{m}$. Therefore, from Lemma 2.4, the germ of the manifold $\left((f, g)\left(\mathbf{R}^{m}\right),(0, g(0))\right)$ is integrable.

5. Examples and counterexamples. Let us define the following four spaces of surface-germs $M=(f, g)\left(\mathbf{R}^{m}\right) \subset T \mathbf{R}^{n}$ involved in our integrability conditions.

$$
\begin{aligned}
\mathcal{M}_{0} & =\{M \mid M \text { satisfies the tangential integrability condition }\} \\
\mathcal{M} & =\{M \mid M \text { integrable }\} \\
\mathcal{M}_{1} & =\left\{M \mid M=(f, g)\left(\mathbf{R}^{m}\right), \text { the linear equation } J f(u) b(u)=g(u)\right. \\
& \text { has a smooth solution }\} \\
\mathcal{M}_{2} & =\{M \mid M \text { is generic in the sense of J. Mather }\} .
\end{aligned}
$$

Proposition 5.1. There is a sequence of proper inclusions

$$
\mathcal{M}_{2} \subset \mathcal{M}_{1} \subset \mathcal{M} \subset \mathcal{M}_{0}
$$

As a proof of this proposition, at each inclusion $\nsubseteq$ we give a simple example which belongs to the latter set but not to the former.

EXAmple 5.1. $M$ fulfils the tangential integrability condition but is not integrable. Let

$$
\begin{gathered}
f, g: \mathbf{R}^{2} \rightarrow \mathbf{R}^{2}, \quad f(u, v)=\left(u, u^{2} v+v^{3}\right), \quad g(u, v)=(1+v, u), \\
M=(f, g)\left(\mathbf{R}^{2}\right) .
\end{gathered}
$$

Then $M$ satisfies the tangential integrability condition

$$
g(u, v) \in J f(u, v)\left(\mathbf{R}^{2}\right),
$$

but $M$ is not integrable at $(1,0)=(f, g)(0,0) \in M$. 
First we easily see that $(f, g): \mathbf{R}^{2} \rightarrow T \mathbf{R}^{2}=\mathbf{R}^{2} \times \mathbf{R}^{2}$ is an embedding. Since

$$
J f(u, v)=\left(\begin{array}{cc}
1 & 0 \\
2 u v & u^{2}+3 v^{2}
\end{array}\right),
$$

at any point $(u, v) \neq(0,0), J f(u, v)$ is a regular matrix and $g(u, v) \in J f(u, v)\left(\mathbf{R}^{2}\right)$. At $(u, v)=(0,0)$,

$$
g(0,0)=\left(\begin{array}{l}
1 \\
0
\end{array}\right) \in\left(\begin{array}{ll}
1 & 0 \\
0 & 0
\end{array}\right) \mathbf{R}^{2} .
$$

Thus $M=(f, g)\left(\mathbf{R}^{2}\right)$ satisfies the tangential integrability condition.

Now let us see that $M$ is not integrable at $(0,0,1,0)=(f, g)(0,0) \in M$. Suppose that $(0,0,1,0)=(f, g)(0,0) \in M$ is an integrable point of $M$. Then there exists an integral curve $\gamma:(-\epsilon, \epsilon) \rightarrow \mathbf{R}^{2}$ such that

$$
\left(\gamma(0), \gamma^{\prime}(0)\right)=(0,0,1,0), \quad\left(\gamma(t), \gamma^{\prime}(t)\right) \in M \text { for all } t \in(-\epsilon, \epsilon) .
$$

Since $(f, g): \mathbf{R}^{2} \rightarrow M \subset T \mathbf{R}^{2}=\mathbf{R}^{2} \times \mathbf{R}^{2}$ is a diffeomorphism, we can consider the curve

$$
\alpha:(-\epsilon, \epsilon) \rightarrow \mathbf{R}^{2} \text { defined by } \alpha(t)=(f, g)^{-1}\left(\gamma(t), \gamma^{\prime}(t)\right) \text {. }
$$

Setting

$$
\alpha(t)=(u(t), v(t)), \quad \text { where } \quad \alpha(0)=(u(0), v(0))=(0,0),
$$

we have the equality

$$
\begin{aligned}
g(\alpha(t))=g(u(t), v(t))=\left(\begin{array}{c}
1+v(t) \\
u(t)
\end{array}\right)= & J f(\alpha(t)) \alpha^{\prime}(t) \\
& =\left(\begin{array}{cc}
1 & 0 \\
2 u(t) v(t) & u(t)^{2}+3 v(t)^{2}
\end{array}\right)\left(\begin{array}{c}
u^{\prime}(t) \\
v^{\prime}(t)
\end{array}\right) .
\end{aligned}
$$

Therefore we have

$$
\begin{aligned}
& 1+v(t)=u^{\prime}(t) \\
& u(t)=2 u(t) v(t) u^{\prime}(t)+\left(u(t)^{2}+3 v(t)^{2}\right) v^{\prime}(t) .
\end{aligned}
$$

Now we compare the orders of both sides of the second equation with respect to the variable $t$. Since $u(0)=v(0)=0$ and from the first equation, we see that

$$
\text { the order of the left hand side } u(t) \text { is equal to } 1 \text {. }
$$

On the other hand, since $u(0)=v(0)=0$, we see that

the order of the right hand side $2 u(t) v(t) u^{\prime}(t)+\left(u(t)^{2}+3 v(t)^{2}\right) v^{\prime}(t)$ is greater than 1 .

This is a contradiction. Thus we see that there is no integral curve $\gamma:(-\epsilon, \epsilon) \rightarrow \mathbf{R}^{2}$ such that

$$
\left(\gamma(0), \gamma^{\prime}(0)\right)=(1,0), \quad\left(\gamma(t), \gamma^{\prime}(t)\right) \in M \text { for all } t \in(-\epsilon, \epsilon),
$$

and $(1,0)=(f, g)(0,0) \in M$ is not an integrable point of $M$.

ExAmple 5.2. $M$ has no smooth solution of the Jacobian linear equation but is integrable. For $n>2$, set

$$
\begin{gathered}
f, g: \mathbf{R}^{2} \rightarrow \mathbf{R}^{2}, \quad f(u, v)=\left(u, v^{n}\right), \quad g(u, v)=(1, v), \\
M=(f, g)\left(\mathbf{R}^{2}\right) .
\end{gathered}
$$


Then $M$ is an integrable manifold, although the Jacobian linear equation $g(u, v)=$ $J f(u, v) b(u)$ has no smooth solution.

First we easily see that $(f, g): \mathbf{R}^{2} \rightarrow T \mathbf{R}^{2}=\mathbf{R}^{2} \times \mathbf{R}^{2}$ is an embedding. Next we check the tangential integrability condition. Since

$$
J f(u, v)=\left(\begin{array}{cc}
1 & 0 \\
0 & n v^{n-1}
\end{array}\right)
$$

at any point $(u, v)$ with $v \neq 0, J f(u, v)$ is a regular matrix and $g(u, v) \in J f(u, v)\left(\mathbf{R}^{2}\right)$. At $(u, 0)$,

$$
g(u, 0)=\left(\begin{array}{l}
1 \\
0
\end{array}\right) \in\left(\begin{array}{ll}
1 & 0 \\
0 & 0
\end{array}\right) \mathbf{R}^{2} .
$$

Thus $M=(f, g)\left(\mathbf{R}^{2}\right)$ satisfies the tangential integrability condition.

Now consider the Jacobian linear equation $g(u, v)=J f(u, v) b(u)$ :

$$
g(u, v)=\left(\begin{array}{l}
1 \\
v
\end{array}\right)=\left(\begin{array}{cc}
1 & 0 \\
0 & n v^{n-1}
\end{array}\right)\left(\begin{array}{l}
b_{1}(u, v) \\
b_{2}(u, v)
\end{array}\right) .
$$

If there exists a smooth solution $\left(b_{1}(u, v), b_{2}(u, v)\right)$, it must satisfy

$$
v=n v^{n-1} b_{2}(u, v) \text {, }
$$

which is impossible for a smooth function $b_{2}(u, v)$ and for $n>2$. Thus the Jacobian linear equation $g(u, v)=J f(u, v) b(u, v)$ has no smooth solutions.

Now let us check that $M$ is integrable. The points $(f, g)(u, v) \in M$ where $v \neq 0$ are integrable, for around the points $(u, v)$ where $v \neq 0$ the Jacobian linear equation $g(u, v)=J f(u, v) b(u, v)$ has smooth solutions.

Now let us check the points $(f, g)(u, 0)=(u, 0,1,0) \in M$. Consider the submanifold

$$
S=\left\{(u, v) \in \mathbf{R}^{2} \mid v=0\right\} \subset \mathbf{R}^{2}
$$

and restrict the Jacobian linear equation to $S$ :

$$
g(u, 0)=\left(\begin{array}{l}
1 \\
0
\end{array}\right)=\left(\begin{array}{ll}
1 & 0 \\
0 & 0
\end{array}\right)\left(\begin{array}{l}
b_{1}(u, 0) \\
b_{2}(u, 0)
\end{array}\right)
$$

Then we have a smooth solution on $S$ :

$$
b_{1}(u, 0)=1, \quad b_{2}(u, 0)=0 .
$$

The solution $\left(b_{1}(u, 0), b_{2}(u, 0)\right)=(1,0)$ is tangent to the submanifold $S$ at every point $(u, 0) \in S$. Consider the integral curves

$$
\alpha(t)=(u+t, 0) \text { of the vector field } \frac{\partial}{\partial u} \text { on } S
$$

and the curves

$$
\gamma: \mathbf{R} \rightarrow \mathbf{R}^{2}, \quad \gamma(t)=f(\alpha(t))=f(u+t, 0)=(u+t, 0) .
$$

Then we have

$$
\begin{gathered}
\left(\gamma(t), \gamma^{\prime}(t)\right)=(u+t, 0,1,0)=(f, g)(u+t, 0) \in(f, g)(S) \subset M, \\
\left(\gamma(0), \gamma^{\prime}(0)\right)=(u, 0,1,0)=(f, g)(u, 0) .
\end{gathered}
$$

Thus the points of the form $\left(\gamma(0), \gamma^{\prime}(0)\right)=(u, 0,1,0)=(f, g)(u, 0)$ are also integrable and hence $M$ is integrable. 
EXAMPLE 5.3. $M$ non-generic but there exists a smooth solution of the Jacobian linear equation. Let

$$
\begin{array}{r}
f, g: \mathbf{R}^{2} \rightarrow \mathbf{R}^{2}, \quad f(u, v)=\left(u, f_{2}(u, v)\right), \quad g(u, v)=\left(1+v,(1+v) \partial f_{2} / \partial u(u, v)\right), \\
\text { such that } \frac{\partial^{2} f_{2}}{\partial u \partial v}(0,0)=\frac{\partial^{2} f_{2}}{\partial v^{2}}(0,0)=0, \\
M=(f, g)\left(\mathbf{R}^{2}\right) .
\end{array}
$$

Then $J f: \mathbf{R}^{2} \rightarrow E(2,2)$ is not transversal to $S_{1}$ but the Jacobian linear equation $g(u, v)=J f(u, v) b(u)$ has a smooth solution, where $E(n, m)$ is the space of $n \times m$ real matrices and $S_{r}$ is the submanifold of $E(n, m)$ consisting of matrices of rank $r$.

First we easily see that $(f, g): \mathbf{R}^{2} \rightarrow T \mathbf{R}^{2}=\mathbf{R}^{2} \times \mathbf{R}^{2}$ is an embedding.

Next let us check that the Jacobian linear equation $g(u, v)=J f(u, v) b(u)$ has a smooth solution. Since

$$
J f(u, v)=\left(\begin{array}{cc}
1 & 0 \\
\partial f_{2} / \partial u(u, v) & \partial f_{2} / \partial v(u, v)
\end{array}\right)
$$

we see that

$$
g(u, v)=\left(\begin{array}{c}
1+v \\
(1+v) \partial f_{2} / \partial u(u, v)
\end{array}\right)=\left(\begin{array}{cc}
1 & 0 \\
\partial f_{2} / \partial u(u, v) & \partial f_{2} / \partial v(u, v)
\end{array}\right)\left(\begin{array}{c}
1+v \\
0
\end{array}\right) .
$$

Thus the Jacobian linear equation $g(u, v)=J f(u, v) b(u)$ has the smooth solution $(1+v, 0)$.

On the other hand, since

$$
\frac{\partial^{2} f_{2}}{\partial u \partial v}(0,0)=\frac{\partial^{2} f_{2}}{\partial v^{2}}(0,0)=0
$$

$J f: \mathbf{R}^{2} \rightarrow E(2,2)$ is not transversal to $S_{1}$ at $(0,0)$, because $J f: \mathbf{R}^{2} \rightarrow E(2,2)$ is transversal to $S_{1}$ at $(0,0)$ if and only if $\partial \operatorname{det} J f / \partial u(0,0) \neq 0$ or $\partial \operatorname{det} J f / \partial v(0,0) \neq 0$.

6. J. Mather's theorem stated in algebraic terms. J. Mather's theorem is stated in geometric terms using the concept of transversality. However if we look more precisely into his proof of the theorem we obtain more weak algebraic condition for the linear equation (2) to have a smooth solution. In order to make our argument simpler we consider our problem in the real analytic category rather than in the $C^{\infty}$ category.

Consider a $n \times m$ matrix $A(x)$ of real analytic function-germs defined at the origin of $\mathbf{R}^{k}$ and a column vector $g(x)$ of $n$ real analytic function-germs defined at the origin of $\mathbf{R}^{k}$.

Consider the linear equation

$$
A(x) b=g(x) .
$$

Let $\mathcal{O}_{k}$ denote the ring of germs at $0 \in \mathbf{R}^{k}$ of real analytic functions of $k$ variables.

First we consider the case $m=n$.

Proposition 6.1. Let $m=n$. If the ideal $\langle\operatorname{det} A(x)\rangle$ in $\mathcal{O}_{k}$ generated by the determinant of the matrix $A(x)$ has property of zeroes (i.e. if any function $h(x)$ vanishes on the variety defined by this ideal then $h(x)$ belongs to the ideal), then the linear equation (5) has a real analytic solution $b(x)$. 
Proof (cf. J. Mather [13], p. 190). Let $\widetilde{A}(x)$ denote the cofactor matrix of $A(x)$. Then we have

$$
\widetilde{A}(x) A(x)=A(x) \widetilde{A}(x)=\operatorname{det} A(x) I_{m},
$$

where $I_{m}$ is the identity matrix of size $m$. Then equation (5) is equivalent to

$$
\operatorname{det} A(x) b=\widetilde{A}(x) g(x) \text {. }
$$

Therefore the component functions $\widetilde{g}_{1}(x), \ldots, \widetilde{g}_{m}(x)$ of the column vector $\widetilde{A}(x) g(x)$ vanish on the variety $\{\operatorname{det} A(x)=0\}$. The hypothesis of Proposition 6.1 implies that $\operatorname{det} A(x)$ divides the component functions $\widetilde{g}_{1}(x), \ldots, \widetilde{g}_{m}(x)$ and

$$
b(x)=\left(\begin{array}{c}
b_{1}(x) \\
\vdots \\
b_{m}(x)
\end{array}\right)=\frac{1}{\operatorname{det} A(x)} \widetilde{A}(x) g(x)=\left(\begin{array}{c}
\widetilde{g}_{1}(x) / \operatorname{det} A(x) \\
\vdots \\
\tilde{g}_{m}(x) / \operatorname{det} A(x)
\end{array}\right),
$$

is a real analytic solution of the equation (5).

REMARK 6.1. In fact one can distinguish three possibilities for the analytic case and $m=n$. Locally, near $0 \in \mathbf{R}^{m}$ we can write $\operatorname{det} A(x)=\phi_{0}(x) \phi_{1}(x)^{k_{1}} \ldots \phi_{r}(x)^{k_{r}}$, where $\phi_{0}(0) \neq 0$ and $\phi_{j}(0)=0$. Now we have three possibilities:

(i) All irreducible components of $C=\{x: \operatorname{det} A(x)=0\}$ defined by $\phi_{j}$ are reduced and of codimension 1 . In this case the system is integrable.

(ii) There is a component of codimension greater than or equal to 2 . In this case we gave Example 5.1 which is related to Bogdanov-Takens singularity (cf. [3]) with the unique separatrix and solution which cannot be analytic.

(iii) There is a non-reduced component of codimension 1. In this non-integrable case one can construct the following example:

$$
f\left(x_{1}, x_{2}\right)=\left(x_{1}, x_{1}^{2} x_{2}-\frac{2}{3} x_{1} x_{2}^{3}+\frac{1}{5} x_{2}^{5}\right), \quad g\left(x_{1}, x_{2}\right)=x_{2} \frac{\partial}{\partial p_{1}}+\left(x_{1}-x_{2}^{2}+\frac{4}{3} x_{2}^{4}\right) \frac{\partial}{\partial p_{2}} .
$$

We have $\operatorname{det} A(x)=\left(x_{1}-x_{2}^{2}\right)^{2}$ and the equation $A b=g$ is reduced to

$$
\left(x_{1}-x_{2}^{2}\right)^{2} \dot{x}_{2}=\left(x_{1}-x_{2}^{2}\right)\left(1-2 x_{2}^{2}\right) .
$$

Thus the tangential integrability condition is satisfied. The phase portrait is given by the equation $\frac{d x_{1}}{d x_{2}}=x_{1}\left(x_{1}-x_{2}^{2}\right) /\left(1-2 x_{2}^{2}\right)$. Near $x=(0,0)$ the phase curves $x_{1}=\psi\left(x_{2}\right)$ through the initial points $\left(x_{2,0}^{2}, x_{2,0}\right) \neq(0,0)$ (i.e. on $C=\left\{x_{1}-x_{2}^{2}=0\right\}$ ) are smooth and transversal to $C$. Hence the equation $\left(x_{1}-x_{2}^{2}\right) \dot{x}_{2}=1-2 x_{2}^{2}$ with $x_{1}=\psi\left(x_{2}\right)=\alpha x_{2}+\ldots$, $\alpha \neq 0$, takes the form $\left(x_{2}-x_{2,0}\right) \dot{x}_{2}=c+\ldots$, with some constant $c \neq 0$. So we have $\left(x_{2}-x_{2,0}\right)(t) \sim$ const. $\sqrt{t}$.

Let us return to the original problem of integrability. We have immediately.

Theorem 6.1. Let $f: \mathbf{R}^{m} \rightarrow \mathbf{R}^{m}$ be a real analytic map and $g: \mathbf{R}^{m} \rightarrow \mathbf{R}^{m}$ be a real analytic map tangent to $f$. If the ideal $\langle\operatorname{det} J f(x)\rangle$ has property of zeroes, then the germ at $(f(0), g(0))$ of $M=(f, g)\left(\mathbf{R}^{m}\right) \subset T \mathbf{R}^{m}$ is integrable.

Now we consider the case $m<n$. Take an $n \times m$ matrix $A(x)$ of real analytic functiongerms defined at the origin of $\mathbf{R}^{k}$ and a column vector $g(x)$ of $n$ real analytic function- 
germs defined at the origin of $\mathbf{R}^{k}$. Consider the equation

$$
A(x) b=g(x)
$$

where $b$ is a column vector of length $m$.

Suppose that the rank of the matrix $A(0)$ at the origin $0 \in \mathbf{R}^{k}$ is $r$. Then we may suppose that the matrix $A(x)$ be of the form

$$
A(x)=\left(\begin{array}{cc}
I_{r} & 0 \\
0 & A^{\prime}(x)
\end{array}\right), \quad \text { where } I_{r} \text { is an identity matrix of size } r
$$

$$
\text { and } A^{\prime}(x)=\left(\begin{array}{cccc}
a_{r+1, r+1}(x) & a_{r+1, r+2}(x) & \ldots & a_{r+1, m}(x) \\
a_{r+2, r+1}(x) & a_{r+2, r+2}(x) & \ldots & a_{r+2, m}(x) \\
\vdots & \vdots & \vdots & \vdots \\
a_{n, r+1}(x) & a_{n, r+2}(x) & \ldots & a_{n, m}(x)
\end{array}\right) .
$$

Consider the set

$$
\mathcal{I}=\left\{I=\left(i_{1}, i_{2}, \ldots, i_{m-r}\right) \mid i_{j} \in \mathbf{N}, r<i_{1}<i_{2}<\ldots<i_{m-r} \leq n\right\},
$$

and for each element $I=\left(i_{1}, i_{2}, \ldots, i_{m-r}\right) \in \mathcal{I}$ consider the $(m-r) \times(m-r)$ minor of $A^{\prime}(x)$ :

$$
\operatorname{det}_{I} A(x)=\operatorname{det}\left(\begin{array}{cccc}
a_{i_{1}, r+1}(x) & a_{i_{1}, r+2}(x) & \cdots & a_{i_{1}, m}(x) \\
a_{i_{2}, r+1}(x) & a_{i_{2}, r+2}(x) & \cdots & a_{i_{2}, m}(x) \\
\vdots & \vdots & \vdots & \vdots \\
a_{i_{m-r}, r+1}(x) & a_{i_{m-r}, r+2}(x) & \cdots & a_{i_{m-r}, m}(x)
\end{array}\right)
$$

Proposition 6.2. If the equation $A(x) b=g(x)$ has a solution $b(x)$ for every $x \in \mathbf{R}^{k}$ and for every element $I \in \mathcal{I}$ the ideal $\left\langle\operatorname{det}_{I} A(x)\right\rangle$ in $\mathcal{O}_{k}$ generated by the $(m-r) \times(m-r)$ minor $\operatorname{det}_{I} A(x)$ of the matrix $A(x)$ has property of zeroes, then the linear equation $A(x) b=g(x)$ has a real analytic solution $b(x)$.

Proof. Although the proof of this proposition is almost the same as the argument in $\S 7$ (pp. 191-192) of J. Mather [13], we recall it here.

First we investigate equation (6) at points $x \in \mathbf{R}^{k}$ where $\operatorname{rank} A(x)=m=\min (m, n)$. Set

$$
\begin{aligned}
& \Omega=\left\{x \in \mathbf{R}^{k} \mid \operatorname{rank} A(x)=m\right\}, \\
& \Sigma=\mathbf{R}^{k}-\Omega=\left\{x \in \mathbf{R}^{k} \mid \operatorname{rank} A(x)<m\right\} .
\end{aligned}
$$

LEMMA 6.1.

1) $\Omega$ is an open dense set of $\mathbf{R}^{k}$.

2) For every point $x \in \Omega$, equation (6) has a unique solution, which is denoted by $b_{\Omega}(x)$.

3) The unique solution $b_{\Omega}(x), x \in \Omega$, is analytic in $\Omega$.

Proof. 1) Is trivial.

2) Since $\operatorname{rank} A(x)=m=\min (m, n)$, if there exists a solution $b(x)$, which follows from the assumption of the lemma, then $b(x)$ is a unique solution of (6). 
3) First note that equation (1) is of the form

$$
\left(\begin{array}{c}
g_{1}(x) \\
\vdots \\
g_{n}(x)
\end{array}\right)=\left(\begin{array}{cc}
I_{r} & 0 \\
0 & A^{\prime}(x)
\end{array}\right)\left(\begin{array}{c}
b_{1}(x) \\
\vdots \\
b_{m}(x)
\end{array}\right) .
$$

Since $x \in \Omega$, there exists $I=\left(i_{1}, i_{2}, \ldots, i_{m-r}\right) \in \mathcal{I}$ such that the submatrix

has rank $m-r$. Set

$$
A_{I}^{\prime}(x)=\left(\begin{array}{cccc}
a_{i_{1}, r+1}(x) & a_{i_{1}, r+2}(x) & \ldots & a_{i_{1}, m}(x) \\
a_{i_{2}, r+1}(x) & a_{i_{2}, r+2}(x) & \ldots & a_{i_{2}, m}(x) \\
\vdots & \vdots & \vdots & \vdots \\
a_{i_{m-r}, r+1}(x) & a_{i_{m-r}, r+2}(x) & \ldots & a_{i_{m-r}, m}(x)
\end{array}\right)
$$

$$
A_{I}(x)=\left(\begin{array}{cc}
I_{r} & 0 \\
0 & A_{I}^{\prime}(x)
\end{array}\right),
$$

and consider the subequation

$$
g_{I}(x)=A_{I}(x) b, \quad \text { where } g_{I}(x)={ }^{t}\left(g_{1}(x), \ldots, g_{r}(x), g_{i_{1}}(x), \ldots, g_{i_{m-r}}(x)\right) .
$$

Since $b_{\Omega}(x)$ is a solution of (6), it is also a solution of the subequation $g_{I}(x)=A_{I}(x) b$, which is analytic since $g_{I}(x)$ is analytic and $\operatorname{det} A_{I}(x) \neq 0$.

Now consider the solution at points $x \in \Sigma$ where $\operatorname{rank} A(x)<m$. Take any $I=$ $\left(i_{1}, i_{2}, \ldots, i_{m-r}\right) \in \mathcal{I}$ and consider the subequation

$$
g_{I}(x)=A_{I}(x) b,
$$

where $A_{I}(x)$ and $g_{I}(x)$ are defined in the same way as those in the proof of Lemma 6.1. Then, from the assumption of Proposition 6.2, the ideal $\left\langle\operatorname{det} A_{I}(x)\right\rangle$ satisfies the condition posed in Proposition 6.1 that it has property of zeroes. Therefore the subequation $g_{I}(x)=$ $A_{I}(x) b$ has an analytic solution $b_{I}(x)$.

Now consider the set

$$
\Omega_{I}=\left\{x \in \mathbf{R}^{k} \mid \operatorname{det} A_{I}(x) \neq 0\right\} \subset \Omega .
$$

Note that $\Omega_{I}$ is also open and dense in $\mathbf{R}^{k}$.

We compare the restriction $\left(\left.b_{I}\right|_{\Omega_{I}}\right)(x)$ of $b_{I}(x)$ to $\Omega_{I}$ with the restriction $\left(\left.b_{\Omega}\right|_{\Omega_{I}}\right)(x)$ of $b_{\Omega}(x)$ to $\Omega_{I}$. Since both $\left(\left.b_{I}\right|_{\Omega_{I}}\right)(x)$ and $\left(\left.b_{\Omega}\right|_{\Omega_{I}}\right)(x)$ are solutions of the subequation

$$
g_{I}(x)=A_{I}(x) b, \quad x \in \Omega_{I},
$$

and the solution of the subequation $g_{I}(x)=A_{I}(x) b$ is unique on the region $\Omega_{I} \subset \Omega$, we have

$$
b_{I}(x)=b_{\Omega}(x) \text { for all } x \in \Omega_{I} .
$$

Now take any other $J \in \mathcal{I}, J \neq I$ and consider the subequation

$$
g_{J}(x)=A_{J}(x) b .
$$

With the same reason, the subequation $g_{J}(x)=A_{J}(x) b$ has an analytic solution $b_{J}(x)$ and we have

$$
b_{J}(x)=b_{\Omega}(x) \quad \text { for all } x \in \Omega_{J} .
$$


Hence we have

$$
b_{J}(x)=b_{I}(x)=b_{\Omega}(x) \quad \text { for all } x \in \Omega_{J} \cap \Omega_{I} .
$$

Thus $b_{J}(x)$ and $b_{I}(x)$ coincide on an open dense subset $\Omega_{J} \cap \Omega_{I}$ of $\mathbf{R}^{k}$ and they are analytic. Therefore they coincide on the whole $\mathbf{R}^{k}$. Set

$$
b(x)=b_{I}(x) \quad \text { for each } I \in \mathcal{I} .
$$

Then $b(x)$ is an analytic solution of all the subequations $g_{I}(x)=A_{I}(x) b$, and it is an analytic solution of the whole equation $g(x)=A(x) b$. Thus Proposition 6.2 is proved.

Now let us go back to the original problem of integrability. We have immediately:

TheOREM 6.2. Let $f: \mathbf{R}^{m} \rightarrow \mathbf{R}^{n}, m<n$, be a real analytic map and $g: \mathbf{R}^{m} \rightarrow \mathbf{R}^{n}$ be a real analytic map tangent to $f$. If for every element $I \in \mathcal{I},\left\langle\operatorname{det}_{I} J f(x)\right\rangle$ has property of zeroes, then the germ of $M=(f, g)\left(\mathbf{R}^{m}\right) \subset T \mathbf{R}^{m}$ at $(f(0), g(0))$ is integrable.

7. Integrability of implicit Hamiltonian systems. Now we consider the special class of implicit differential systems - the generalized Hamiltonian systems, which play an important role in field theory and mechanics.

Let $\left(\mathbf{R}^{2 n}, \omega\right)$ be the symplectic Euclidean space, endowed with the symplectic structure in the Darboux form, $\omega=\sum_{i=1}^{n} d y_{i} \wedge d x_{i}$. By the vector bundle morphism $\beta$ : $\left.T \mathbf{R}^{2 n} \ni u \rightarrow u\right\rfloor \omega \in T^{*} \mathbf{R}^{2 n}$ we introduce the canonical symplectic structure $\bar{\omega}$ on $T \mathbf{R}^{2 n}$, namely the pullback of the Liouville symplectic form $d \theta$ defined on $T^{*} \mathbf{R}^{2 n}$ :

$$
\bar{\omega}=\beta^{*} d \theta=\sum_{i=1}^{n}\left(d \dot{y}_{i} \wedge d x_{i}-d \dot{x}_{i} \wedge d y_{i}\right)
$$

where $(p, q)=((x, y),(\dot{x}, \dot{y}))$ are coordinates on $T \mathbf{R}^{2 n} \equiv \mathbf{R}^{2 n} \times \mathbf{R}^{2 n}$.

Definition 7.1. A smooth submanifold $M \subset T \mathbf{R}^{2 n} \equiv \mathbf{R}^{2 n} \times \mathbf{R}^{2 n}$ is called a generalized Hamiltonian system if $M$ is isotropic according to $\bar{\omega}$, i.e. $\left.\bar{\omega}\right|_{M}=0$. In this case $\operatorname{dim} M \leq 2 n$. The case of $M$ such that $\operatorname{dim} M=2 n$ is called the implicit Hamiltonian system.

By the Hörmander-Arnold-Weinstein generating family representation of $M$ [1], we have the following lemma.

Lemma 7.1. Let $M \subset T \mathbf{R}^{2 n} \equiv \mathbf{R}^{2 n} \times \mathbf{R}^{2 n}$ be an implicit Hamiltonian system, and let $(p, q) \in M$. Suppose that corank $d\left(\left.\pi_{1}\right|_{M}\right)(p, q)=k$. Then there exist an open neighbourhood $\mathcal{O}$ of $(p, q)$ in $T \mathbf{R}^{2 n}$ and a smooth function $F: \mathbf{R}^{2 n} \times \mathbf{R}^{k} \rightarrow \mathbf{R}$ defined on some open neighbourhood of $(p, 0)$, such that

$$
\begin{array}{r}
M \cap \mathcal{O}=\left\{(p, q) \in \mathcal{O} \mid \exists \lambda \in \mathbf{R}^{k} \text { such that } \dot{x}_{i}=\frac{\partial F}{\partial y_{i}}(x, y, \lambda),\right. \\
\left.\dot{y}_{j}=-\frac{\partial F}{\partial x_{j}}(x, y, \lambda), 0=\frac{\partial F}{\partial \lambda_{l}}(x, y, \lambda)\right\},
\end{array}
$$

where $1 \leq i, j \leq n, 1 \leq l \leq k$, and that

$$
\operatorname{rank}\left(\frac{\partial^{2} F}{\partial x_{j} \partial \lambda_{l}}, \frac{\partial^{2} F}{\partial y_{i} \partial \lambda_{l}}, \frac{\partial^{2} F}{\partial \lambda_{s} \partial \lambda_{l}}\right)(p, 0)=k, \quad \text { and } \quad \frac{\partial^{2} F}{\partial \lambda_{s} \partial \lambda_{r}}(p, 0)=0 \text {, }
$$

where $1 \leq s, r \leq k$. 
In what follows we will consider the Hamiltonian case, i.e. $\operatorname{dim} M=2 n$, and because we are considering the local properties of $M$, we assume that $M$ is generated by some generating family-germ $F$ as in Lemma 7.1 and denote it also by $M_{F}$.

LEMMA 7.2. If $M_{F} \subset T \mathbf{R}^{2 n} \equiv \mathbf{R}^{2 n} \times \mathbf{R}^{2 n}$ is an implicit Hamiltonian system generated by the Morse family-germ $F:\left(\mathbf{R}^{2 n} \times \mathbf{R}^{k},(0,0)\right) \rightarrow \mathbf{R}$, then the tangential integrability condition of $M_{F}$ is equivalent to the existence of the solution $\mu=\left(\mu_{1}, \ldots, \mu_{n}\right) \in \mathbf{R}^{k}$ of the equation

$$
\left(\begin{array}{ccc}
\frac{\partial^{2} F}{\partial \lambda_{1} \partial \lambda_{1}} & \cdots & \frac{\partial^{2} F}{\partial \lambda_{1} \partial \lambda_{k}} \\
\vdots & \vdots & \vdots \\
\frac{\partial^{2} F}{\partial \lambda_{k} \partial \lambda_{1}} & \cdots & \frac{\partial^{2} F}{\partial \lambda_{k} \partial \lambda_{k}}
\end{array}\right)\left(\begin{array}{c}
\mu_{1} \\
\vdots \\
\mu_{k}
\end{array}\right)=\left(\begin{array}{c}
\left\{\frac{\partial F}{\partial \lambda_{1}}, F\right\} \\
\vdots \\
\left\{\frac{\partial F}{\partial \lambda_{k}}, F\right\}
\end{array}\right)
$$

for each $(p, q) \in M$, where $\{\cdot, \cdot\}$ denotes the Poisson bracket on $\mathbf{R}^{2 n}$ induced by $\omega$.

Proof. Assume that $\gamma:(-\varepsilon, \varepsilon) \rightarrow \mathbf{R}^{2 n}$ and $\delta:(-\varepsilon, \varepsilon) \rightarrow \mathbf{R}^{k}, \gamma(0)=(x, y), \delta(0)=\lambda$, is a smooth solution of $(7)$, through the point $(x, y, \lambda)$ of $M$. Then we have

$$
\frac{\partial F}{\partial \lambda_{l}}(\gamma(t), \delta(t)) \equiv 0, \quad t \in(-\varepsilon, \varepsilon), 1 \leq l \leq k
$$

and also

$$
\begin{gathered}
\sum_{i=1}^{k} \frac{\partial^{2} F}{\partial \lambda_{i} \partial \lambda_{l}}(\gamma(t), \delta(t)) \delta_{i}^{\prime}(t)+\sum_{i=1}^{k} \frac{\partial^{2} F}{\partial x_{i} \partial \lambda_{l}}(\gamma(t), \delta(t)) \dot{x}_{i}+\sum_{i=1}^{k} \frac{\partial^{2} F}{\partial y_{i} \partial \lambda_{l}}(\gamma(t), \delta(t)) \dot{y}_{i} \\
=\sum_{i=1}^{k} \frac{\partial^{2} F}{\partial \lambda_{i} \partial \lambda_{l}}(\gamma(t), \delta(t)) \delta_{i}^{\prime}(t)+\sum_{i=1}^{k} \frac{\partial^{2} F}{\partial x_{i} \partial \lambda_{l}}(\gamma(t), \delta(t)) \frac{\partial F}{\partial y_{i}}(\gamma(t), \delta(t)) \\
-\sum_{i=1}^{k} \frac{\partial^{2} F}{\partial y_{i} \partial \lambda_{l}}(\gamma(t), \delta(t)) \frac{\partial F}{\partial x_{i}}(\gamma(t), \delta(t)), \quad 1 \leq l \leq k
\end{gathered}
$$

Taking $\gamma(0)=(x, y), \delta(0)=\lambda$, we get the solvability of the equation

$$
\sum_{i=1}^{k} \frac{\partial^{2} F}{\partial \lambda_{i} \partial \lambda_{l}}(x, y, \lambda) \mu_{i}-\left\{\frac{\partial F}{\partial \lambda_{l}}, F\right\}=0, \quad 1 \leq l \leq k
$$

on the smooth manifold given by the equations $\frac{\partial F}{\partial \lambda_{l}}(x, y, \lambda)=0$.

The tangential integrability condition is the necessary condition for integrability of $M$. Now we give an example of implicit Hamiltonian system which fulfils the tangential integrability condition but it is not integrable.

EXAmple 7.1. As in the case of Example 5.1, we show that the tangential integrability condition is also only a necessary condition for implicit Hamiltonian systems.

Let us consider the implicit Hamiltonian system surface-germ $M \subset T \mathbf{R}^{2} \equiv \mathbf{R}^{2} \times \mathbf{R}^{2}$ around $(p, q)=(0,0,1,0)$. $M$ is generated by the family-germ $F: \mathbf{R}^{2} \times \mathbf{R}^{2} \rightarrow \mathbf{R}$, at $\left(x, y, \lambda_{1}, \lambda_{2}\right)=(0,0,1,0)$ of the form

$$
F(x, y, \lambda)=\frac{1}{2} \lambda_{2}^{2}\left(1-\left(\lambda_{1}-1\right)^{2}\right)-\frac{1}{4}\left(\lambda_{1}-1\right)^{4}+\lambda_{1} y-\lambda_{2} x .
$$


So one can write the corresponding equations for $M$ in the form

$$
\begin{aligned}
\dot{x} & =\frac{\partial F}{\partial y}(x, y, \lambda)=\lambda_{1}, \\
\dot{y} & =-\frac{\partial F}{\partial x}(x, y, \lambda)=\lambda_{2}, \\
0 & =\frac{\partial F}{\partial \lambda_{1}}(x, y, \lambda)=y-\lambda_{2}^{2}\left(\lambda_{1}-1\right)-\left(\lambda_{1}-1\right)^{3}, \\
0 & =\frac{\partial F}{\partial \lambda_{2}}(x, y, \lambda)=-x+\lambda_{2}\left(1-\left(\lambda_{1}-1\right)^{2}\right) .
\end{aligned}
$$

Certainly tangential integrability condition is fulfilled around $(0,0,1,0)$. The determinant of the matrix

$$
\left(\begin{array}{cc}
\frac{\partial^{2} F}{\partial \lambda_{1} \partial \lambda_{1}} & \frac{\partial^{2} F}{\partial \lambda_{1} \partial \lambda_{2}} \\
\frac{\partial^{2} F}{\partial \lambda_{2} \partial \lambda_{1}} & \frac{\partial^{2} F}{\partial \lambda_{2} \partial \lambda_{2}}
\end{array}\right)(x, y, \lambda)
$$

has isolated zero at $(0,0,1,0)$ and so there exists a solution of the linear equation

$$
\left(\begin{array}{cc}
\frac{\partial^{2} F}{\partial \lambda_{1} \partial \lambda_{1}} & \frac{\partial^{2} F}{\partial \lambda_{1} \partial \lambda_{2}} \\
\frac{\partial^{2} F}{\partial \lambda_{2} \partial \lambda_{1}} & \frac{\partial^{2} F}{\partial \lambda_{2} \partial \lambda_{2}}
\end{array}\right)\left(\begin{array}{l}
\mu_{1} \\
\mu_{2}
\end{array}\right)=\left(\begin{array}{c}
\left\{\frac{\partial F}{\partial \lambda_{1}}, F\right\} \\
\left\{\frac{\partial F}{\partial \lambda_{2}}, F\right\}
\end{array}\right)
$$

in some neighbourhood outside of $(0,0,1,0)$. At that point we have

$$
\left(\begin{array}{ll}
0 & 0 \\
0 & 1
\end{array}\right)\left(\begin{array}{l}
\mu_{1} \\
\mu_{2}
\end{array}\right)=\left(\begin{array}{l}
0 \\
1
\end{array}\right)
$$

and it is also solvable. So the tangential integrability condition is fulfilled.

If $M$ is integrable, say at $(p, q)=(0,0,1,0)$, then there exists an integral curve $\gamma:(-\varepsilon, \varepsilon) \rightarrow \mathbf{R}^{2}$ such that

$$
\left(\gamma(0), \gamma^{\prime}(0)\right)=(0,0,1,0) \quad \text { and } \quad\left(\gamma(t), \gamma^{\prime}(t)\right) \in M \text { for } t \in(-\varepsilon, \varepsilon) .
$$

So there exists a smooth curve $\lambda(t)=\left(\lambda_{1}(t), \lambda_{2}(t)\right), \lambda(0)=(1,0)$ such that

$$
\left(\begin{array}{cc}
\frac{\partial^{2} F}{\partial \lambda_{1} \partial \lambda_{1}} & \frac{\partial^{2} F}{\partial \lambda_{1} \partial \lambda_{2}} \\
\frac{\partial^{2} F}{\partial \lambda_{2} \partial \lambda_{1}} & \frac{\partial^{2} F}{\partial \lambda_{2} \partial \lambda_{2}}
\end{array}\right)(\gamma(t), \lambda(t))\left(\begin{array}{c}
\lambda_{1}^{\prime} \\
\lambda_{2}^{\prime}
\end{array}\right)=\left(\begin{array}{c}
-\lambda_{2}(t) \\
\lambda_{1}(t)
\end{array}\right)
$$

i.e. the following equations are fulfilled:

$$
\begin{aligned}
& \lambda_{2}(t)=3\left(\lambda_{1}(t)-1\right)^{2} \lambda_{1}^{\prime}(t)+\lambda_{2}^{2}(t) \lambda_{1}^{\prime}(t)+2 \lambda_{2}(t)\left(\lambda_{1}(t)-1\right) \lambda_{2}^{\prime}(t) \\
& \lambda_{1}(t)=\left(1-\left(\lambda_{1}(t)-1\right)^{2}\right) \lambda_{2}^{\prime}(t)-2 \lambda_{2}(t)\left(\lambda_{1}(t)-1\right) \lambda_{1}^{\prime}(t) .
\end{aligned}
$$

From equation (11) we get

$$
\lambda_{2}^{\prime}(0)=1
$$

so from the first equation (10) we have a contradiction. Namely the order of $\lambda_{2}(t)$ at 0 is equal to 1 , but the order of

$$
3\left(\lambda_{1}(t)-1\right)^{2} \lambda_{1}^{\prime}(t)+\lambda_{2}^{2}(t) \lambda_{1}^{\prime}(t)+2 \lambda_{2}(t)\left(\lambda_{1}(t)-1\right) \lambda_{2}^{\prime}(t)
$$

at 0 is greater than or equal to 2 . 
Let $\tilde{M}_{F} \subset T \mathbf{R}^{2 n} \times \mathbf{R}^{k} \equiv \mathbf{R}^{2 n} \times \mathbf{R}^{2 n} \times \mathbf{R}^{k}$ denote a manifold-germ defined by equations (7) in Lemma 7.1. Consider the projection

$$
\pi: \mathbf{R}^{2 n} \times \mathbf{R}^{2 n} \times \mathbf{R}^{k} \rightarrow \mathbf{R}^{2 n} \times \mathbf{R}^{k}, \quad(p, q, \lambda) \rightarrow(p, \lambda),
$$

and the image $C_{F}=\pi\left(\tilde{M}_{F}\right)$, which is a smooth manifold-germ by transversality condition (8) in Lemma 7.1.

Let

$$
H=\left(\begin{array}{ccc}
\frac{\partial^{2} F}{\partial \lambda_{1} \partial \lambda_{1}}(x, y, \lambda) & \cdots & \frac{\partial^{2} F}{\partial \lambda_{1} \partial \lambda_{k}}(x, y, \lambda) \\
\vdots & \ddots & \vdots \\
\frac{\partial^{2} F}{\partial \lambda_{k} \partial \lambda_{1}}(x, y, \lambda) & \cdots & \frac{\partial^{2} F}{\partial \lambda_{k} \partial \lambda_{k}}(x, y, \lambda)
\end{array}\right)
$$

By

$$
\tilde{H}=\left(\begin{array}{ccc}
{\left[\frac{\partial^{2} F}{\partial \lambda_{1} \partial \lambda_{1}}(x, y, \lambda)\right]} & \cdots & {\left[\frac{\partial^{2} F}{\partial \lambda_{1} \partial \lambda_{k}}(x, y, \lambda)\right]} \\
\vdots & \ddots & \vdots \\
{\left[\frac{\partial^{2} F}{\partial \lambda_{k} \partial \lambda_{1}}(x, y, \lambda)\right]} & \cdots & {\left[\frac{\partial^{2} F}{\partial \lambda_{k} \partial \lambda_{k}}(x, y, \lambda)\right]}
\end{array}\right)
$$

we denote the $k \times k$ cofactor matrix of $H$ in $(p, q) \in M_{F}$. Then, by Cramer's rule equation (9) can be rewritten in the form $(\operatorname{det} H) \mu=\tilde{H}\left\{\frac{\partial F}{\partial \lambda}, F\right\}$, i.e.

$$
(\operatorname{det} H)(x, y, \lambda) \mu_{i}=\sum_{j=1}^{k}\left[\frac{\partial^{2} F}{\partial \lambda_{i} \partial \lambda_{j}}\right]\left\{\frac{\partial F}{\partial \lambda_{j}}, F\right\}(x, y, \lambda) \text {. }
$$

So we have an equivalent formulation of the tangential integrability condition.

Corollary 7.1. If $M_{F} \subset T \mathbf{R}^{2 n} \equiv \mathbf{R}^{2 n} \times \mathbf{R}^{2 n}$ is an implicit Hamiltonian system generated by the Morse family-germ $F:\left(\mathbf{R}^{2 n} \times \mathbf{R}^{k},(0,0)\right) \rightarrow \mathbf{R}$, then the tangential integrability condition of $M_{F}$ is equivalent to the condition

$$
\left.\sum_{j=1}^{k}\left[\frac{\partial^{2} F}{\partial \lambda_{i} \partial \lambda_{j}}\right]\left\{\frac{\partial F}{\partial \lambda_{j}}, F\right\}\right|_{\{\operatorname{det} H=0\} \cap C_{F}} \equiv 0 .
$$

Now we have the result corresponding to Lemma 2.4.

Lemma 7.3. Let $F: \mathbf{R}^{2 n} \times \mathbf{R}^{k} \rightarrow \mathbf{R}$ be the smooth generating family for $M_{F}$. If the linear equation

$$
\left(\begin{array}{ccc}
\frac{\partial^{2} F}{\partial \lambda_{1} \partial \lambda_{1}}(x, y, \lambda) & \cdots & \frac{\partial^{2} F}{\partial \lambda_{1} \partial \lambda_{k}}(x, y, \lambda) \\
\vdots & \ddots & \vdots \\
\frac{\partial^{2} F}{\partial \lambda_{k} \partial \lambda_{1}}(x, y, \lambda) & \cdots & \frac{\partial^{2} F}{\partial \lambda_{k} \partial \lambda_{k}}(x, y, \lambda)
\end{array}\right)\left(\begin{array}{c}
\mu_{1}(x, y, \lambda) \\
\vdots \\
\mu_{k}(x, y, \lambda)
\end{array}\right)=\left(\begin{array}{c}
\left\{\frac{\partial F}{\partial \lambda_{1}}, F\right\}(x, y, \lambda) \\
\vdots \\
\left.\frac{\partial F}{\partial \lambda_{k}}, F\right\}(x, y, \lambda)
\end{array}\right)
$$

has a smooth solution $\left(\mu_{1}(x, y, \lambda), \ldots, \mu_{k}(x, y, \lambda)\right)$ on the critical manifold

$$
C_{F}=\left\{(x, y, \lambda) \mid \frac{\partial F}{\partial \lambda_{i}}(x, y, \lambda)=0,0 \leq i \leq k\right\},
$$

then the implicit Hamiltonian system $M_{F}$ is integrable. 
Proof. We can easily see, on the basis of Lemma 7.1, that if we find the smooth solution of the linear equation (9) with respect to $\mu$, then the system of equations

$$
\begin{aligned}
& \dot{x}_{i}=\frac{\partial F}{\partial y_{i}}(x, y, \lambda), \quad 1 \leq i \leq n, \\
& \dot{y}_{j}=-\frac{\partial F}{\partial x_{j}}(x, y, \lambda), \quad 1 \leq j \leq n, \\
& \dot{\lambda}_{l}=\mu_{l}(x, y, \lambda), \quad 1 \leq l \leq k,
\end{aligned}
$$

is locally integrable over the surface $\left\{(x, y, \lambda) \mid \frac{\partial F}{\partial \lambda_{l}}(x, y, \lambda)=0,1 \leq l \leq k\right\}$.

Problem 7.1. Find conditions to be posed on a smooth generating family $F: \mathbf{R}^{2 n} \times$ $\mathbf{R}^{k} \rightarrow \mathbf{R}$, so that the linear equation (9) has a smooth solution on $C_{F}$.

Let $E_{s}(k, k)$ denote the space of $k \times k$ symmetric matrices of real numbers. For each integer $r \geq 0$ let $S_{r}$ denote the subset of $E_{s}(k, k)$ consisting of all symmetric matrices of rank $r$. Then $\Sigma_{r}$ is a submanifold of $E_{s}(k, k)$ of codimension $(k-r)(k-r+1) / 2$. Now we have a well defined mapping of $M_{F}$ into symmetric matrices $E_{s}(k, k)$. Because the projections $\left.\pi_{T \mathbf{R}^{2 n}}\right|_{\tilde{M}_{F}}: \tilde{M}_{F} \rightarrow M_{F}, \pi_{T \mathbf{R}^{2 n}}: T \mathbf{R}^{2 n} \times \mathbf{R}^{k} \rightarrow T \mathbf{R}^{2 n}$ and $\left.\pi\right|_{\tilde{M}_{F}}: \tilde{M}_{F} \rightarrow C_{F}$ are embeddings, then we can uniquely represent this mapping by $H: C_{F} \rightarrow E_{s}(k, k)$,

$$
\left.H(x, y, \lambda)\right|_{\left\{(x, y, \lambda) \in C_{F}\right\}}=\left(\begin{array}{ccc}
\frac{\partial^{2} F}{\partial \lambda_{1} \partial \lambda_{1}}(x, y, \lambda) & \cdots & \frac{\partial^{2} F}{\partial \lambda_{1} \partial \lambda_{k}}(x, y, \lambda) \\
\vdots & \ddots & \vdots \\
\frac{\partial^{2} F}{\partial \lambda_{k} \partial \lambda_{1}}(x, y, \lambda) & \cdots & \frac{\partial^{2} F}{\partial \lambda_{k} \partial \lambda_{k}}(x, y, \lambda)
\end{array}\right)
$$

Definition 7.2. An implicit Hamiltonian system $M_{F} \subset T \mathbf{R}^{2 n} \equiv \mathbf{R}^{2 n} \times \mathbf{R}^{2 n}$, generated by the generating family $F: \mathbf{R}^{2 n} \times \mathbf{R}^{k} \rightarrow \mathbf{R}$ is called generic if the map $H: C_{F} \rightarrow E_{s}(k, k)$ is transversal to all $S_{r}, r=0, \ldots, k-1$.

Now we can formulate the main result of this section.

THEOREM 7.1. The generic implicit Hamiltonian system $M \subset T \mathbf{R}^{2 n}$ is integrable if and only if it satisfies the tangential integrability condition.

Proof. The proof of this theorem follows from J. Mather's theorem (cf. [13], p. 185) concerning the smooth solvability of linear equations with symmetric matrix.

Using results of Section 6 we can formulate the corresponding results concerning integrability of implicit Hamiltonian systems. Now our function-matrix $\left.H\right|_{\left\{(x, y, \lambda) \in C_{F}\right\}}$ : $C_{F} \rightarrow E_{s}(k, k)$ corresponds to the matrix $A(x)$ in Proposition 6.1. Let $\mathcal{O}_{C_{F}, 0}$ denote the ring of germs at $0 \in C_{F}$ of real analytic functions on $C_{F}$. Then on the basis of Proposition 6.1 and Theorem 6.1 we get the following result.

Theorem 7.2. Let $F:\left(\mathbf{R}^{2 n} \times \mathbf{R}^{k}, 0\right) \rightarrow \mathbf{R}$ be a real analytic function-germ. Let $M_{F}$ generated by $F$ fulfil the tangential integrability condition. If the ideal $\left\langle\operatorname{det}\left(\left.H\right|_{C_{F}}\right)(x, y, \lambda)\right\rangle$ in $\mathcal{O}_{C_{F}, 0}$ has property of zeroes, then the germ at $(0,0)$ of $M_{F}$ is integrable. 
A class of implicit Hamiltonian systems for which the tangential integrability condition is also the sufficient condition is provided by the generating family-germs of the form

$$
F(x, y, \lambda)=f(x, y)+\sum_{i=1}^{k} \lambda_{i} f_{i}(x, y)
$$

where $\tilde{f}(x, y)=\left(f_{1}(x, y), \ldots, f_{k}(x, y)\right), 1 \leq k \leq n$ is a non-singular smooth map-germ $\left(\mathbf{R}^{2 n}, 0\right) \rightarrow\left(\mathbf{R}^{k}, 0\right)$. Thus $M_{F}$, called the constrained Hamiltonian system, is a smooth Lagrangian submanifold-germ over the submanifold $K=\tilde{f}^{-1}(0)$.

Now the tangential integrability condition from the Lemma 7.2 becomes the system of equations

$$
\left.\left\{\frac{\partial F}{\partial \lambda_{i}}, F\right\}\right|_{\tilde{f}^{-1}(0)}=0, \quad 1 \leq i \leq k .
$$

This system is equivalent to

$$
\begin{gathered}
\left.\left\{f_{i}(x, y), f_{j}(x, y)\right\}\right|_{\tilde{f}^{-1}(0)}=0, \quad 1 \leq i, j \leq k, \\
\left.\left\{f(x, y), f_{l}(x, y)\right\}\right|_{\tilde{f}^{-1}(0)}=0, \quad 1 \leq l \leq k,
\end{gathered}
$$

which in fact defines $K$ to be a coisotropic submanifold of $\left(\mathbf{R}^{2 n}, \omega\right)$, i.e. geometrically $T_{q} K \supset\left\{u \in T_{q} \mathbf{R}^{2 n} \mid \omega(u, v)=0 \forall v \in T_{q} K\right\}$, and $f$ restricts to those which are constant on the leaves of the characteristic foliation of $K$. Now we have the integrability result proved also in geometric way in ([16], Theorem 8.1).

Corollary 7.2. Let $M_{F}$ be a constrained Hamiltonian system over the submanifold $K \subset \mathbf{R}^{2 n}$ and $F$ fulfil the tangential integrability condition. Then $K$ is a coisotropic submanifold of $\left(\mathbf{R}^{2 n}, \omega\right)$ and $M_{F}$ is integrable.

8. Non-integrable points are isolated generically in Tougeron's sense. In this section we will investigate the non-integrable points. We prove that non-transversal points, i.e. the points where $J f: \mathbf{R}^{m} \rightarrow E(n, m)$ is not transversal to $S_{r}$, are generically isolated, which implies that non-integrable points are generically isolated.

Here, in this section, the genericity notion is in the strongest sense introduced by J. P. Tougeron [23]:

Definition 8.1. We say that the non-transversal points of $M=(f, g)\left(\mathbf{R}^{m}\right)$ are generically isolated if there exists an $\infty$-codimensional subset $\Sigma_{\infty}\left(\mathbf{R}^{m}, \mathbf{R}^{n}\right)$ of the space $C^{\infty}\left(\mathbf{R}^{m}, \mathbf{R}^{n}\right)$ of $C^{\infty}$ mappings of $\mathbf{R}^{m}$ into $\mathbf{R}^{n}$ such that for any $f \in C^{\infty}\left(\mathbf{R}^{m}, \mathbf{R}^{n}\right)-$ $\Sigma_{\infty}\left(\mathbf{R}^{m}, \mathbf{R}^{n}\right)$ non-transversal points are isolated.

Let us now fix the notation. Set

$$
\begin{gathered}
C^{\infty}\left(\mathbf{R}^{m}, T \mathbf{R}^{n}\right)=\left\{(f, g): \mathbf{R}^{m} \rightarrow \mathbf{R}^{n} \times \mathbf{R}^{n}=T \mathbf{R}^{n} \mid(f, g) \text { is a } C^{\infty} \text { mapping }\right\} \\
\quad=C^{\infty}\left(\mathbf{R}^{m}, \mathbf{R}^{n}\right) \times C^{\infty}\left(\mathbf{R}^{m}, \mathbf{R}^{n}\right) \\
T\left(\mathbf{R}^{m}, T \mathbf{R}^{n}\right)=\left\{(f, g): \mathbf{R}^{m} \rightarrow \mathbf{R}^{n} \times \mathbf{R}^{n}=T \mathbf{R}^{n} \mid g \text { is tangent to } f\right\},
\end{gathered}
$$

where all these spaces are endowed with Whitney $C^{\infty}$ topology. Recall that $g$ is tangent to $f$ if and only if $(f, g)$ is an embedding and $M=(f, g)\left(\mathbf{R}^{m}\right)$ satisfies the tangential integrability condition (see Definition 3.2). 
Definition 8.2. Let $(f, g) \in T\left(\mathbf{R}^{m}, T \mathbf{R}^{n}\right)$. A point $(x, \dot{x})=(f(u), g(u)) \in M=$ $(f, g)\left(\mathbf{R}^{m}\right)$ is a transversal point of $M$ if $J f: \mathbf{R}^{m} \rightarrow E(n, m)$ is transversal to $S_{r}$ at $u \in \mathbf{R}^{m}$ for $r=\operatorname{rank} J f(u)$, equivalently $j^{1}\left(\left.\pi_{1}\right|_{M}\right): M \rightarrow J^{1}\left(M, \mathbf{R}^{n}\right)$ is transversal to

$$
S_{r}\left(M, \mathbf{R}^{n}\right)=\left\{j^{1} h(v) \in J^{1}\left(M, \mathbf{R}^{n}\right) \mid \operatorname{rank} J h(v)=r\right\},
$$

where $\pi_{1}: T \mathbf{R}^{n}=\mathbf{R}^{n} \times \mathbf{R}^{n} \rightarrow \mathbf{R}^{n}$ is the projection defined by $\pi_{1}(x, \dot{x})=x$.

We are interested in the structure of the non-generic (in the sense of the previous sections) points and to investigate how they are located in $M$. We prove the following main theorems.

TheOREM 8.1. There exists an $\infty$-codimensional subset $\Sigma_{\infty} T\left(\mathbf{R}^{m}, T \mathbf{R}^{n}\right)$ of the set $T\left(\mathbf{R}^{m}, T \mathbf{R}^{n}\right)$ such that for any embedding

$$
(f, g) \in T\left(\mathbf{R}^{m}, T \mathbf{R}^{n}\right)-\Sigma_{\infty} T\left(\mathbf{R}^{m}, T \mathbf{R}^{n}\right)
$$

non-transversal points of $M=(f, g)\left(\mathbf{R}^{m}\right)$ are isolated.

Combining this theorem with J. Mather's theorem, we obtain

TheOREM 8.2. Let $\Sigma_{\infty} T\left(\mathbf{R}^{m}, T \mathbf{R}^{n}\right)$ be the $\infty$-codimensional subset of $T\left(\mathbf{R}^{m}, T \mathbf{R}^{n}\right)$ given in Theorem 8.1. Then for any

$$
(f, g) \in T\left(\mathbf{R}^{m}, T \mathbf{R}^{n}\right)-\Sigma_{\infty} T\left(\mathbf{R}^{m}, T \mathbf{R}^{n}\right)
$$

non-integrable points of $M=(f, g)\left(\mathbf{R}^{m}\right)$ are isolated.

REMARK 8.1. Let us define the set

$$
\Sigma_{\mathrm{tr}} T\left(\mathbf{R}^{m}, T \mathbf{R}^{n}\right)=\left\{(f, g) \in T\left(\mathbf{R}^{m}, T \mathbf{R}^{n}\right) \mid J f: U \rightarrow E(n, m)\right.
$$

is not transversal to $S_{r}$ for some $r$.

Then

$$
\Sigma_{\infty} T\left(\mathbf{R}^{m}, T \mathbf{R}^{n}\right) \subset \Sigma_{\operatorname{tr}} T\left(\mathbf{R}^{m}, T \mathbf{R}^{n}\right),
$$

$\operatorname{codim} \Sigma_{\mathrm{tr}} T\left(\mathbf{R}^{m}, T \mathbf{R}^{n}\right)$ in $T\left(\mathbf{R}^{m}, T \mathbf{R}^{n}\right)$ is less than $\infty$,

$$
\text { codim } \Sigma_{\infty} T\left(\mathbf{R}^{m}, T \mathbf{R}^{n}\right) \text { in } T\left(\mathbf{R}^{m}, T \mathbf{R}^{n}\right) \text { equals } \infty \text {. }
$$

This means that even if $M=(f, g)\left(\mathbf{R}^{m}\right)$ is not a generic submanifold in the sense of Section 3, non-transversal points and non-integrable points of $M=(f, g)\left(\mathbf{R}^{m}\right)$ are isolated points for a very generic $(f, g)$, i.e. for any $(f, g) \in \Sigma_{\operatorname{tr}} T\left(\mathbf{R}^{m}, T \mathbf{R}^{n}\right)-\Sigma_{\infty} T\left(\mathbf{R}^{m}, T \mathbf{R}^{n}\right)$.

For the strong genericity result we need an appropriate transversality theorem. Let us define

$$
\begin{aligned}
J^{r}(m, n) & =\left\{j^{r} f(0) \mid f \in C^{\infty}\left(\mathbf{R}^{m}, \mathbf{R}^{n}\right), \quad f(0)=0\right\} \\
J^{r}\left(\mathbf{R}^{m}, \mathbf{R}^{n}\right) & =\left\{j^{r} f(u) \mid f \in C^{\infty}\left(\mathbf{R}^{m}, \mathbf{R}^{n}\right), u \in \mathbf{R}^{m}\right\} .
\end{aligned}
$$

For positive integers $s>r$, we define the canonical projections

$$
\pi_{r}^{s}: J^{s}(m, n) \rightarrow J^{r}(m, n), \quad \pi_{r}^{s}\left(j^{s} f(0)\right)=j^{r} f(0) .
$$

TheOrem 8.3 (Transversality). Let $W$ be a semi-algebraic subset of $J^{r}(m, n)$ and let $X_{1}, \ldots, X_{\ell}$ be a finite number of semi-algebraic submanifolds of $J^{k}\left(\mathbf{R}^{m}, \mathbf{R}^{n}\right)$. Then there 
exists a closed semi-algebraic subset

$\Sigma_{W}$ of $\left(\pi_{r}^{r+k+1}\right)^{-1}(W)$ having codimension greater than or equal to 1 such that for any $C^{\infty}$ mapping $f: \mathbf{R}^{m} \rightarrow \mathbf{R}^{n}$ with

$$
j^{r+k+1}(0) \in\left(\pi_{r}^{r+k+1}\right)^{-1}(W)-\Sigma_{W},
$$

there exists a neighbourhood $U$ of the origin 0 of $\mathbf{R}^{m}$ such that $j^{r} f: U-\{0\} \rightarrow$ $J^{k}\left(\mathbf{R}^{m}, \mathbf{R}^{n}\right)$ is transversal to $X_{1}, \ldots, X_{\ell}$ at every point $u \in U$ except at the origin $0 \in \mathbf{R}^{m}$.

For the proof we refer to the more general version of Theorem 8.3 which is proved in [8] (Theorem 1, p. 229).

8.1. Proof of Theorem 8.1. Let us identify $J^{1}(m, n)$ with $E(n, m)$ and set

$$
\begin{aligned}
S_{r}\left(\mathbf{R}^{m}, \mathbf{R}^{n}\right)=\left\{j^{1} f(u)\right. & \left.\in J^{1}\left(\mathbf{R}^{m}, \mathbf{R}^{n}\right) \mid \operatorname{rank} J f(u)=r\right\} \\
& =\mathbf{R}^{m} \times \mathbf{R}^{n} \times S_{r} \subset \mathbf{R}^{m} \times \mathbf{R}^{n} \times J^{1}(m, n)=J^{1}\left(\mathbf{R}^{m}, \mathbf{R}^{n}\right) .
\end{aligned}
$$

For $\left(u_{0}, x_{0}\right) \in \mathbf{R}^{m} \times \mathbf{R}^{n}$, set

$$
C^{\infty}\left(\mathbf{R}^{m}, \mathbf{R}^{n} ; u_{0}, x_{0}\right)=\left\{f \in C^{\infty}\left(\mathbf{R}^{m}, \mathbf{R}^{n}\right) \mid f\left(u_{0}\right)=x_{0}\right\} .
$$

We prove Theorem 8.1 using the following result:

THEOREM 8.4. There exists an infinite-codimensional subset $\Sigma_{\infty}\left(\mathbf{R}^{m}, \mathbf{R}^{n} ; u_{0}, x_{0}\right)$ of $C^{\infty}\left(\mathbf{R}^{m}, \mathbf{R}^{n} ; u_{0}, x_{0}\right)$ such that for any $C^{\infty}$ mapping $f: \mathbf{R}^{m} \rightarrow \mathbf{R}^{n}$ with

$$
f \in C^{\infty}\left(\mathbf{R}^{m}, \mathbf{R}^{n} ; u_{0}, x_{0}\right)-\Sigma_{\infty}\left(\mathbf{R}^{m}, \mathbf{R}^{n} ; u_{0}, x_{0}\right)
$$

there exists a neighbourhood $V\left(u_{0}\right)$ of $u_{0}$ in $\mathbf{R}^{m}$ such that $j^{1} f: V\left(u_{0}\right)-\left\{u_{0}\right\} \rightarrow$ $J^{1}\left(\mathbf{R}^{m}, \mathbf{R}^{n}\right)$ is transversal to $S_{r}\left(\mathbf{R}^{m}, \mathbf{R}^{n}\right)$ for $r=0, \ldots, \min (m, n)$ at every point $u \in$ $V\left(u_{0}\right)-\left\{u_{0}\right\}$.

Proof of Theorem 8.1. Set

$$
\begin{aligned}
\Sigma_{\infty}\left(\mathbf{R}^{m}, \mathbf{R}^{n}\right) & =\underset{(u, x) \in \mathbf{R}^{m} \times \mathbf{R}^{n}}{ } \Sigma_{\infty}\left(\mathbf{R}^{m}, \mathbf{R}^{n} ; u, x\right) \subset C^{\infty}\left(\mathbf{R}^{m}, \mathbf{R}^{n}\right) \\
\Sigma_{\infty}\left(\mathbf{R}^{m}, T \mathbf{R}^{n}\right) & =\left\{(f, g) \in C^{\infty}\left(\mathbf{R}^{m}, T \mathbf{R}^{n}\right) \mid f \in \Sigma_{\infty}\left(\mathbf{R}^{m}, \mathbf{R}^{n}\right)\right\} \\
& =\Sigma_{\infty}\left(\mathbf{R}^{m}, \mathbf{R}^{n}\right) \times C^{\infty}\left(\mathbf{R}^{m}, \mathbf{R}^{n}\right) \\
\Sigma_{\infty} T\left(\mathbf{R}^{m}, T \mathbf{R}^{n}\right) & =\Sigma_{\infty}\left(\mathbf{R}^{m}, T \mathbf{R}^{n}\right) \cap T\left(\mathbf{R}^{m}, T \mathbf{R}^{n}\right),
\end{aligned}
$$

where we note that $T \mathbf{R}^{n}=\mathbf{R}^{n} \times \mathbf{R}^{n}$ and hence that

$$
C^{\infty}\left(\mathbf{R}^{m}, T \mathbf{R}^{n}\right)=C^{\infty}\left(\mathbf{R}^{m}, \mathbf{R}^{n}\right) \times C^{\infty}\left(\mathbf{R}^{m}, \mathbf{R}^{n}\right) .
$$

We claim that $\Sigma_{\infty} T\left(\mathbf{R}^{m}, T \mathbf{R}^{n}\right)$ is the required subset.

It is easy to see that if $(f, g) \in T\left(\mathbf{R}^{m}, T \mathbf{R}^{n}\right)-\Sigma_{\infty} T\left(\mathbf{R}^{m}, T \mathbf{R}^{n}\right)$, then non-transversal points of $M=(f, g)\left(\mathbf{R}^{m}\right)$ are isolated.

Now we investigate the codimension of this set. Since $\Sigma_{\infty}\left(\mathbf{R}^{m}, \mathbf{R}^{n} ; u_{0}, x_{0}\right)$ is $\infty$ codimensional in $C^{\infty}\left(\mathbf{R}^{m}, \mathbf{R}^{n} ; u_{0}, x_{0}\right)$, so is $\Sigma_{\infty}\left(\mathbf{R}^{m}, \mathbf{R}^{n}\right)$ in $C^{\infty}\left(\mathbf{R}^{m}, \mathbf{R}^{n}\right)$. Therefore $\operatorname{codim} \Sigma_{\infty}\left(\mathbf{R}^{m}, \mathbf{R}^{n}\right) \times C^{\infty}\left(\mathbf{R}^{m}, \mathbf{R}^{n}\right)$ in $C^{\infty}\left(\mathbf{R}^{m}, T \mathbf{R}^{n}\right)$ is equal to $\infty$. 
Define

$$
\begin{gathered}
T_{0}\left(\mathbf{R}^{m}, T \mathbf{R}^{n}\right)=\left\{(f, g) \in C^{\infty}\left(\mathbf{R}^{m}, T \mathbf{R}^{n}\right) \mid g(u) \in J f(u)\left(\mathbf{R}^{m}\right) \forall u \in \mathbf{R}^{m}\right\}, \\
\operatorname{Emb}\left(\mathbf{R}^{m}, T \mathbf{R}^{n}\right)=\left\{(f, g) \in C^{\infty}\left(\mathbf{R}^{m}, T \mathbf{R}^{n}\right) \mid(f, g) \text { is an embedding }\right\} .
\end{gathered}
$$

Then we have

$$
T\left(\mathbf{R}^{m}, T \mathbf{R}^{n}\right)=\operatorname{Emb}\left(\mathbf{R}^{m}, T \mathbf{R}^{n}\right) \cap T_{0}\left(\mathbf{R}^{m}, T \mathbf{R}^{n}\right)
$$

Since

$$
\Sigma_{\infty}\left(\mathbf{R}^{m}, T \mathbf{R}^{n}\right)=\Sigma_{\infty}\left(\mathbf{R}^{m}, \mathbf{R}^{n}\right) \times C^{\infty}\left(\mathbf{R}^{m}, \mathbf{R}^{n}\right)
$$

and

$\operatorname{codim} \Sigma_{\infty}\left(\mathbf{R}^{m}, \mathbf{R}^{n}\right) \times C^{\infty}\left(\mathbf{R}^{m}, \mathbf{R}^{n}\right)$ in $C^{\infty}\left(\mathbf{R}^{m}, T \mathbf{R}^{n}\right)$ is equal to $\infty$,

we have

$\operatorname{codim} \Sigma_{\infty}\left(\mathbf{R}^{m}, T \mathbf{R}^{n}\right) \cap T_{0}\left(\mathbf{R}^{m}, T \mathbf{R}^{n}\right)$ in $T_{0}\left(\mathbf{R}^{m}, T \mathbf{R}^{n}\right)$ is equal to $\infty$.

Since

$$
\operatorname{Emb}\left(\mathbf{R}^{m}, T \mathbf{R}^{n}\right) \text { is open in } C^{\infty}\left(\mathbf{R}^{m}, T \mathbf{R}^{n}\right)
$$

we see that

$$
\begin{aligned}
\operatorname{codim} \Sigma_{\infty}\left(\mathbf{R}^{m}, T \mathbf{R}^{n}\right) \cap T_{0}\left(\mathbf{R}^{m}, T \mathbf{R}^{n}\right) \cap \operatorname{Emb}\left(\mathbf{R}^{m}, T \mathbf{R}^{n}\right) \\
\\
\text { in } T_{0}\left(\mathbf{R}^{m}, T \mathbf{R}^{n}\right) \cap \operatorname{Emb}\left(\mathbf{R}^{m}, T \mathbf{R}^{n}\right) \text { is equal to } \infty
\end{aligned}
$$

Since

$$
\begin{aligned}
\Sigma_{\infty} T\left(\mathbf{R}^{m}, T \mathbf{R}^{n}\right) & =\Sigma_{\infty}\left(\mathbf{R}^{m}, T \mathbf{R}^{n}\right) \cap T\left(\mathbf{R}^{m}, T \mathbf{R}^{n}\right) \\
& =\Sigma_{\infty}\left(\mathbf{R}^{m}, T \mathbf{R}^{n}\right) \cap T_{0}\left(\mathbf{R}^{m}, T \mathbf{R}^{n}\right) \cap \operatorname{Emb}\left(\mathbf{R}^{m}, T \mathbf{R}^{n}\right) \\
T\left(\mathbf{R}^{m}, T \mathbf{R}^{n}\right) & =T_{0}\left(\mathbf{R}^{m}, T \mathbf{R}^{n}\right) \cap \operatorname{Emb}\left(\mathbf{R}^{m}, T \mathbf{R}^{n}\right)
\end{aligned}
$$

we see that

$$
\operatorname{codim} \Sigma_{\infty} T\left(\mathbf{R}^{m}, T \mathbf{R}^{n}\right) \text { in } T\left(\mathbf{R}^{m}, T \mathbf{R}^{n}\right) \text { is equal to } \infty .
$$

This completes the proof of Theorem 8.1.

Proof of Theorem 8.4. It suffices to prove Theorem 8.4 for $\left(u_{0}, x_{0}\right)=(0,0)$, so we reformulate it in an accessible way.

THEOREM 8.4'. There exists an infinite-codimensional subset $\Sigma_{\infty}\left(\mathbf{R}^{m}, \mathbf{R}^{n} ; 0,0\right)$ of $C^{\infty}\left(\mathbf{R}^{m}, \mathbf{R}^{n} ; 0,0\right)$ such that for any $C^{\infty}$ mapping $f: \mathbf{R}^{m} \rightarrow \mathbf{R}^{n}$ with

$$
f \in C^{\infty}\left(\mathbf{R}^{m}, \mathbf{R}^{n} ; 0,0\right)-\Sigma_{\infty}\left(\mathbf{R}^{m}, \mathbf{R}^{n} ; 0,0\right)
$$

there exists a neighbourhood $V(0)$ of 0 in $\mathbf{R}^{m}$ such that $j^{1} f: V(0)-\{0\} \rightarrow J^{1}\left(\mathbf{R}^{m}, \mathbf{R}^{n}\right)$ is transversal to $S_{r}\left(\mathbf{R}^{m}, \mathbf{R}^{n}\right)$ for $r=0, \ldots, \min (m, n)$ at every point $u \in V(0)-\{0\}$.

Proof. First we apply Theorem 8.3 to $W=W_{1}=J^{1}(m, n)$ and

$$
X_{r}=S_{r}\left(\mathbf{R}^{m}, \mathbf{R}^{n}\right) \subset J^{1}\left(\mathbf{R}^{m}, \mathbf{R}^{n}\right), \quad r=0, \ldots, \min (m, n) .
$$

Then there exists a closed semi-algebraic subset

$\Sigma_{1}$ of $\left(\pi_{1}^{1+1+1}\right)^{-1}(W)=J^{3}(m, n)$ having codimension greater than or equal to 1 
such that for any $C^{\infty}$ mapping $f: \mathbf{R}^{m} \rightarrow \mathbf{R}^{n}$ with

$$
j^{3}(0) \in J^{3}(m, n)-\Sigma_{1},
$$

there exists a neighbourhood $U$ of the origin 0 of $\mathbf{R}^{m}$ such that

$$
j^{1} f: U-\{0\} \rightarrow J^{1}\left(\mathbf{R}^{m}, \mathbf{R}^{n}\right) \text { is transversal to } S_{r}\left(\mathbf{R}^{m}, \mathbf{R}^{n}\right) \text { for each } r .
$$

Next we apply Theorem 8.3 to $W=\Sigma_{1} \subset J^{3}(m, n)$ and

$$
X_{r}=S_{r}\left(\mathbf{R}^{m}, \mathbf{R}^{n}\right) \subset J^{1}\left(\mathbf{R}^{m}, \mathbf{R}^{n}\right), \quad r=0, \ldots, \min (m, n) .
$$

Then, again there exists a closed semi-algebraic subset

$$
\Sigma_{2} \text { of }\left(\pi_{3}^{3+1+1}\right)^{-1}\left(\Sigma_{1}\right) \subset J^{5}(m, n)
$$

such that

$$
\begin{gathered}
\text { codimension of } \Sigma_{2} \text { in }\left(\pi_{3}^{5}\right)^{-1}\left(\Sigma_{1}\right) \text { is greater than } 0, \\
\text { codimension of } \Sigma_{2} \text { in } J^{5}(m, n) \text { is greater than } 1 \text {, }
\end{gathered}
$$

and such that for any $C^{\infty}$ mapping $f: \mathbf{R}^{m} \rightarrow \mathbf{R}^{n}$ with

$$
j^{5}(0) \in J^{5}(m, n)-\Sigma_{2},
$$

there exists a neighbourhood $U$ of the origin 0 of $\mathbf{R}^{m}$ such that

$$
j^{1} f: U-\{0\} \rightarrow J^{1}\left(\mathbf{R}^{m}, \mathbf{R}^{n}\right) \text { is transversal to } S_{r}\left(\mathbf{R}^{m}, \mathbf{R}^{n}\right) \text { for each } r .
$$

In this way, we can prove inductively that there exists a closed semi-algebraic subset

$$
\Sigma_{k} \text { of } J^{2 k+1}(m, n) \text { with codimension greater than or equal to } k
$$

such that for any $C^{\infty}$ mapping $f: \mathbf{R}^{m} \rightarrow \mathbf{R}^{n}$ with

$$
j^{2 k+1}(0) \in J^{2 k+1}(m, n)-\Sigma_{k},
$$

there exists a neighbourhood $U$ of the origin 0 of $\mathbf{R}^{m}$ such that

$$
j^{1} f: U-\{0\} \rightarrow J^{1}\left(\mathbf{R}^{m}, \mathbf{R}^{n}\right) \text { is transversal to } S_{r}\left(\mathbf{R}^{m}, \mathbf{R}^{n}\right) \text { for each } r .
$$

Set

$$
\begin{gathered}
\Sigma_{k}\left(\mathbf{R}^{m}, \mathbf{R}^{n} ; 0,0\right)=\left\{f \in C^{\infty}\left(\mathbf{R}^{m}, \mathbf{R}^{n} ; 0,0\right) \mid j^{2 k+1}(0) \in \Sigma_{k}\right\}, \\
\Sigma_{\infty}\left(\mathbf{R}^{m}, \mathbf{R}^{n} ; 0,0\right)=\bigcap_{k=1}^{\infty} \Sigma_{k}\left(\mathbf{R}^{m}, \mathbf{R}^{n} ; 0,0\right) .
\end{gathered}
$$

Then $\Sigma_{\infty}\left(\mathbf{R}^{m}, \mathbf{R}^{n} ; 0,0\right)$ satisfies the condition required in Theorem 8.4.

This completes the proof of Theorem 8.4 and hence also the proof of Theorem 8.1.

Acknowledgments. The authors thank the referee for helpful comments. This paper was written during the second author's stay at the Max Planck Institute of Mathematics in Bonn. 


\section{References}

[1] V. I. Arnol'd, S. M. Gusel̆n-Zade, A. N. Varchenko, Singularities of Differentiable Maps I, Monogr. Math. 82, Birkhäuser, Boston, 1985.

[2] J. Basto-Gonçalves, Implicit Hamiltonian equations, Mat. Contemp. 12 (1997), 123-137.

[3] R. I. Bogdanov, Local orbital normal forms of vector fields on a plane, Trudy Sem. Petrovsk. 5 (1979), 51-84 (in Russian).

[4] J. W. Bruce, A note on first order differential equations of degree greater than one and wavefront evolution, Bull. London Math. Soc. 16 (1984), 139-144.

[5] A. Davydov, Normal form for a differential equation, not solvable for the derivative, in a neighbourhood of a singular point, Funktsional. Anal. i Prilozhen. 19:2 (1985), 1-10 (in Russian); English transl.: Funct. Anal. Appl. 19 (1985), 81-89.

[6] P. A. M. Dirac, Generalized Hamiltonian dynamics, Canadian J. Math. 2 (1950), 129-148.

[7] I. Ekeland, Discontinuités de champs hamiltoniens et existence de solutions optimales en calcul des variations, Inst. Hautes Études Sci. Publ. Math. 47 (1977), 5-32.

[8] T. Fukuda, Local topological properties of differentiable mappings I, Invent. Math. 65 (1981), $227-250$.

[9] C. G. Gibson, K. Wirthmüller, A. A. du Plessis, E. J. N. Looijenga, Topological Stability of Smooth Mappings, Lecture Notes in Math. 552, Springer, Berlin, 1976.

[10] S. Janeczko, On implicit Lagrangian differential systems, Ann. Polon. Math. 74 (2000), 133-141.

[11] W. Liu, H. J. Sussmann, Shortest paths for sub-Riemannian metrics on rank-two distributions, Mem. Amer. Math. Soc. 118, no. 564, Amer. Math. Soc., Providence, 1995.

[12] J. Martinet, Singularities of Smooth Functions and Maps, London Math. Soc. Lecture Note Ser. 58, Cambridge Univ. Press, Cambridge, 1982.

[13] J. N. Mather, Solutions of generic linear equations, in: Dynamical Systems (Proc. Sympos., Univ. Bahia, 1971), Academic Press, New York, 185-193.

[14] J. N. Mather, Stability of $C^{\infty}$ mappings. V. Transversality, Advances in Math. 4 (1970), 301-336.

[15] G. Mendella, G. Marmo, W. M. Tulczyjew, Integrability of implicit differential equations, J. Phys. A 28 (1995), 149-163.

[16] M. R. Menzio, W. M. Tulczyjew, Infinitesimal symplectic relations and generalized Hamiltonian dynamics, Ann. Inst. H. Poincaré Sect. A (N.S.) 28 (1978), 349-367.

[17] B. Morin, Formes canoniques des singularités d'une application différentiable, C. R. Acad. Sci. Paris 260 (1965), 5662-5665.

[18] R. Narasimhan, Introduction to the Theory of Analytic Spaces, Lecture Notes in Math. 25, Springer, Berlin, 1966.

[19] L. S. Pontryagin, V. G. Boltyanskii, R. V. Gamkrelidze, E. F. Mishchenko, The Mathematical Theory of Optimal Processes, Pergamon Press, New York, 1964.

[20] J. Sotomayor, M. Zhitomirskii, Impasse singularities of differential systems of the form $A(x) x^{\prime}=F(x)$, J. Differential Equations 169 (2001), 567-587.

[21] F. Takens, Implicit differential equations: some open problems, in: Singularités d'applications différentiables, Lecture Notes in Math. 535, Springer, Berlin, 1976, 237-253.

[22] R. Thom, Sur les équations différentielles multiforms et leurs intégrales singuliérs, Colloque E. Cartan, Paris, 1971.

[23] J. C. Tougeron, Idéaux de fonctions différentiables I, Ann. Inst. Fourier (Grenoble) 18 (1986), 177-240.

[24] A. Weinstein, Lectures on Symplectic Manifolds, CBMS Regional Conf. Ser. in Math. 29, Amer. Math. Soc., Providence, 1977. 